\title{
Why is sterility virulence most common in sexually transmitted infections? Examining the role of epidemiology
}

\author{
David V. McLeod*1 and Troy Day ${ }^{\dagger 2}$ \\ ${ }^{1}$ Institute of Integrative Biology, ETH Zürich, Zürich, Switzerland \\ ${ }^{2}$ Department of Mathematics and Statistics, Department of Biology Queen's \\ University, Kingston, ON, Canada
}

Author Contributions: DVM and TD designed study, analyzed model, and wrote paper.

Acknowledgments: Financial support was provided by an ETH Zürich postdoctoral fellowship (to DVM). All simulations were run on the HPC cluster at ETH Zürich.

Keywords: infectious disease; virulence evolution; sterility; stochasticity; sexuallytransmitted infections Data associated with this manuscript can be found at https://datadryad.org/resource/ doi:10.5061/dryad.70423j5

*david.mcleod@env.ethz.ch

†day@queensu.ca

This article has been accepted for publication and undergone full peer review but has not been through the copyediting, typesetting, pagination and proofreading process, which may lead to differences between this version and the Version of Record. Please cite this article as doi: $10.1111 /$ evo. 13718

This article is protected by copyright. All rights reserved. 


\begin{abstract}
Sterility virulence, or the reduction in host fecundity due to infection, occurs in many host-pathogen systems. Notably, sterility virulence is more common for sexuallytransmitted infections (STIs) than for directly-transmitted pathogens, while other forms of virulence tend to be limited in STIs. This has led to the suggestion that sterility virulence may have an adaptive explanation. By focusing upon finite population models, we show that the observed patterns of sterility virulence can be explained by consideration of the epidemiological differences between STIs and directlytransmitted pathogens. In particular, when pathogen transmission is predominantly density-invariant (as for STIs), and mortality is density-dependent, sterility virulence can be favoured by demographic stochasticity, whereas if pathogen transmission is predominantly density-dependent, as is common for most directly-transmitted pathogens, sterility virulence is disfavoured. We show these conclusions can hold even if there is a weak selective advantage to sterilizing.
\end{abstract}

This article is protected by copyright. All rights reserved. 


\section{Introduction}

2 Sterility virulence, defined to be any reduction in host fecundity due to pathogen infection

3 (including complete sterility), occurs in many host-pathogen systems (Abbate et al. 2015;

4 Apari et al. 2014; Lockhart et al. 1996) but is more commonly associated with sexually-

5 transmitted infections (STIs; Lockhart et al. 1996). Interestingly, sterilizing STIs tend to

6 cause limited virulence other than sterility (Antonovics et al. 2011; Lockhart et al. 1996),

whereas sterilizing non-STIs tend to be associated with high overall levels of virulence (Apari

et al. 2014). In combination, this evidence has led to the suggestion that sterility virulence

9 may be a 'targeted' pathogen strategy with an adaptive explanation (Apari et al. 2014),

rather than simply a physiological by-product of STIs being localized to the reproductive organs (Antonovics et al. 2011). In particular, it has been hypothesized that by causing host reproductive failure, sterility virulence may promote host sexual activity and thus increase STI transmission (Apari et al. 2014). However, this explanation requires individuals to have both the ability to detect reproductive failure (e.g., a species with long-term pair bonds) and the capacity to act upon this information (e.g., divorce and find new mate), and so the hypothesis is primarily targeted towards humans (Apari et al. 2014). Yet as Apari et al. (2014) noted, sterilizing STIs are neither restricted to humans nor species with long-term pair bonds (Knell and Webberley 2004; Lockhart et al. 1996; Smith and Dobson 1992), limiting the generality of this explanation. For example, the two-spot ladybird and koala are both highly promiscuous with no long-term pair bonds, yet experience high rates of infection by sterilizing STIs (Hurst et al. 1995; Weigler et al. 1988).

One key difference between STIs and other directly transmitted pathogens is the epidemiology of transmission. For pathogens transmitted by direct (non-sexual) contact, transmission is generally density-dependent as contacts between individuals tend to increase with density (Anderson and May 1979; Begon et al. 2002; McCallum et al. 2017). For STIs, however, transmission tends to be mainly frequency-dependent as sex acts and reproduction are largely determined by mating system, and only weakly depend upon population density in the 
absence of Allee effects (Anderson and May 1991; Antonovics et al. 2011; Lloyd-Smith et al. 2004; Lockhart et al. 1996; May and Anderson 1987). Although the form of transmission has clear epidemiological consequences (de Castro and Bolker 2005; Getz and Pickering 1983), in deterministic models with large (ideally infinite) population sizes, if sterility virulence causes any increase in transmissibility, sterilizing pathogens are favoured, irrespective of the form of transmission (Jaenike 1996; O'Keefe 2005; O'Keefe and Antonovics 2002). Indeed, evolutionary theory on sterility virulence has focused almost exclusively upon such models (Ashby and Boots 2015; Best et al. 2010; Jaenike 1996; Lion and Gandon 2015; McLeod and Day 2015; O'Keefe and Antonovics 2002), ignoring the evolutionary consequences of epidemiology in finite populations. Instead these previous studies have focused upon how spatial structure (Lion and Gandon 2015; O'Keefe and Antonovics 2002) or host co-evolution (Ashby and Boots 2015; Best et al. 2010; McLeod and Day 2015) can limit the evolution of sterility virulence, without considering why sterility virulence is more common for STIs than directly-transmitted pathogens.

Here we consider how the interaction between epidemiology and demographic stochasticity can explain the observed patterns of sterility virulence in finite populations. Our analysis shows that when transmission is mainly density-invariant and infected individuals experience some density-dependent mortality, pathogen strains causing some degree of sterility tend to be favoured. If instead transmission is mainly density-dependent or infected individuals do not experience density-dependent mortality, then pathogen strains causing no sterility virulence are favoured. Hence the interaction between epidemiology and demographic stochasticity can help explain why sterility virulence is more commonly associated with STIs than directly-transmitted pathogens. To explore the robustness of our results to the inclusion of the possibility of other selective effects of sterility virulence, we also consider the possibility of a (weak) selective advantage to sterility virulence (i.e., transmissibility increases with sterility virulence). When this occurs, both demographic stochasticity and selection favour sterility virulence in STIs. However, for directly-transmitted pathogens sterility virulence is disfavoured by demographic stochasticity but favoured by selection. We 
provide the conditions under which demographic stochasticity nevertheless overcomes selection to disfavour sterility virulence in directly transmitted pathogens. Taken as a whole, our results provide a novel explanation for why sterility virulence is more commonly seen in STIs, and why directly-transmitted pathogens should not cause sterility, even when favoured to by selection.

\section{Model}

Consider a susceptible-infected host population with horizontal transmission, no multiple infection and no recovery. Let the density of susceptible hosts and hosts infected with strain $i=1,2$ at time $t$ be $x(t)$ and $y_{i}(t)$, respectively, and take $\mathbf{x}=\left(x, y_{1}, y_{2}\right)$. Susceptible and infected hosts die at a per capita rate of $d(\mathbf{x})$ and $\mu(\mathbf{x})$, respectively, and mortality is a nondecreasing function of population density $\left(\partial d / \partial \mathbf{x}_{i} \geq 0, \partial \mu / \partial \mathbf{x}_{i} \geq 0\right)$. Susceptible hosts are born at rate $b(\mathbf{x})$, which may be regulated by density-dependent processes (e.g., logistic growth), while the fecundity of a host infected with strain $i$ is reduced by a factor of $1-\delta_{i}$ due to sterility virulence: if $\delta_{i}=1$, sterility virulence is maximal and strain $i$ fully sterilizes the host, whereas if $\delta_{i}=0$, strain $i$ causes no sterility virulence. Thus if strain $i$ is present in the population, $\partial b / \partial \delta_{i}<0$. The per capita transmission rate of strain $i$ is $\beta_{i}(\mathbf{x})$ and due to pathogen mutation (and strain replacement at the within-host level), strain $i$ infected hosts transition to strain $j$ infected hosts at a per capita rate $\nu$; as this is assumed to be rare, $\nu \ll 1$. For brevity, we will refer to $\nu$ as mutation rate. We will assume strain 1 causes less host sterility than strain $2, \delta_{1}<\delta_{2}$, but may have reduced transmissibility, $\beta_{1}(\mathbf{x})=\beta(\mathbf{x})(1-\varepsilon), \beta_{2}(\mathbf{x})=\beta(\mathbf{x})$, and that selection on sterility virulence through transmission is weak, $0 \leq \varepsilon \ll 1$. Note that the assumption of weak selection places no restrictions upon the between-strain difference in sterility virulence, so in the most extreme case, it is possible one strain fully sterilizes while the other causes no sterility virulence, that is, $\delta_{1}=0, \delta_{2}=1$.

Under these assumptions, the population dynamics can be described using the following 
system of stochastic differential equations (SDEs)

$$
\begin{aligned}
d x & =\left[b(\mathbf{x})-d(\mathbf{x}) x-\beta_{1}(\mathbf{x}) y_{1}-\beta_{2}(\mathbf{x}) y_{2}\right] d t+\Omega^{-1 / 2} M_{x} \\
d y_{1} & =\left[\left(\beta_{1}(\mathbf{x})-\mu(\mathbf{x})\right) y_{1}+\nu\left(y_{2}-y_{1}\right)\right] d t+\Omega^{-1 / 2} M_{y_{1}} \\
d y_{2} & =\left[\left(\beta_{2}(\mathbf{x})-\mu(\mathbf{x})\right) y_{2}+\nu\left(y_{1}-y_{2}\right)\right] d t+\Omega^{-1 / 2} M_{y_{2}}
\end{aligned}
$$

where $\Omega$ is habitat size, and so controls the likelihood of interactions between hosts, regardless of infection status, while $M_{x}, M_{y_{i}}$ are unbiased stochastic noise terms. Critically, these noise terms arise naturally from the individual birth, death, and transmission events from the full stochastic process underlying the approximation given by (1), and so are dependent upon the population demographics in a well-defined fashion (see SI 1 for the full derivation). As habitat size becomes large $(\Omega \rightarrow \infty)$ we will be left with a system of ordinary differential equations (ODEs). In the absence of mutations $(\nu=0)$, the ODE system predicts the strain with higher transmissibility (strain 2) will fix in the population, irrespective of the form of the transmission function, $\beta(\mathbf{x})$, or the between-strain difference in sterility virulence. This can be seen by considering the conditions under which strain $i$ can invade a monomorphic strain $j$ population at equilibrium: from (1), strain $i$ will have a positive per capita growth when rare if $\beta_{i}(\mathbf{x})>\mu(\mathbf{x})$. Since at the monomorphic strain $j$ equilibrium we have $\beta_{j}(\mathbf{x})=$ $\mu(\mathbf{x})$, at equilibrium the condition $\beta_{i}(\mathbf{x})>\mu(\mathbf{x})$ becomes $\beta_{i}(\mathbf{x})>\beta_{j}(\mathbf{x})$, and so whichever strain has the higher transmissibility is favoured. If instead the two strains have the same transmissibility, but different sterility virulence, then in the ODE model with no mutation

$$
\frac{d y_{1} / d t}{d y_{2} / d t}=\frac{(\beta(\mathbf{x})-\mu(\mathbf{x})) y_{1}}{(\beta(\mathbf{x})-\mu(\mathbf{x})) y_{2}}=\frac{y_{1}}{y_{2}}
$$

and so it follows that $y_{1}=C_{0} y_{2}$, that is, the density of strain 1 is a linear function of the density of strain 2, with the slope determined by the initial state of the population at $t=0$, that is, $C_{0}=y_{1}(0) / y_{2}(0)$. It follows that the fraction of infected hosts that are infected by strain 1 will remain constant over time and is set by the initial state of the population at $t=0$, that is, $\frac{y_{1}(t)}{y_{1}(t)+y_{2}(t)}=\frac{y_{1}(0)}{y_{1}(0)+y_{2}(0)}$, and so the between-strain difference in sterility 
virulence is selectively neutral with respect to the deterministic evolutionary dynamics.

Because system (1) is a set of coupled SDEs, as currently written it does not provide much insight into the evolutionary process. Therefore we wish to reduce it to a more tractable form. To do so, we observe that since selection is weak and mutations rare, system (1) admits what is commonly referred to as a 'slow manifold' (Berglund and Gentz 2006; Parsons and Rogers 2017), which in this case is a curve in $\left(x, y_{1}, y_{2}\right)$-space satisfying $\beta(\mathbf{x})=\mu(\mathbf{x})$. The reason that this curve is referred to as a slow manifold is that in the vicinity of this curve, the per capita growth rate of the different pathogen strains is very small (since mutations are rare and selection is weak), and so changes in the composition of the pathogen population occur slowly. As a result, system (1) has a fast timescale and a slow timescale (Fig. 1). The fast timescale corresponds to demographic processes (e.g., transmission, birth, and death events) driven mainly by the terms in (1) multiplied by $d t$; the effect of these processes is to push the system into the vicinity of the slow manifold. In this fast timescale regime, stochasticity has a weak effect, and the dynamics of (1) are very similar to the dynamics of the set of ordinary differential equations (obtained in the limit of $\Omega \rightarrow \infty$; see Fig. 1). Once in the vicinity of the slow manifold, however, the system slowly moves along the slow manifold as the composition of the pathogen population changes due to mutation, selection and stochasticity (this is the slow timescale; see Fig. 1).

As our interest is the evolution of the pathogen, we are primarily interested in the dynamics on the slow timescale, and so we wish to transform system (1) to a simpler form in which the fast timescale dynamics are eliminated. Let $p \equiv y_{1} /\left(y_{1}+y_{2}\right)$ be the proportion of infected hosts that are infected with strain 1. Then using methods outlined in recent work (Constable et al. 2016; Parsons and Rogers 2017), which built off of standard techniques (Berglund and Gentz 2006; Katzenberg 1991), the slow timescale dynamics of $p$ are governed by the stochastic differential equation (SDE)

$$
d p=\underbrace{\left(\nu(1-2 p)-\varepsilon \beta\left(\mathbf{x}_{p}\right) p(1-p)\right)}_{a(p)} d t+(\underbrace{\frac{p(1-p)}{\Omega} \mathfrak{R}\left(\mathbf{x}_{p}\right)}_{\sigma^{2}(p)})^{1 / 2} d W_{t}
$$



$(1)$

where $d W_{t}$ is a Gaussian random variable with mean zero and variance $d t$ (see SI 2 for details). In $(3), \mathbf{x}_{p}=\left[x_{p}, p \mathcal{I}(p),(1-p) \mathcal{I}(p)\right]$ is the vector of population densities evaluated on the slow timescale, so $x_{p}$ and $\mathcal{I}(p)$ are the density of susceptible hosts and total density of infected hosts, respectively, on the slow timescale for a given $p$, and

$$
\mathfrak{R}\left(\mathbf{x}_{p}\right)=\frac{\beta\left(\mathbf{x}_{p}\right)+\mu\left(\mathbf{x}_{p}\right)}{\mathcal{I}(p)}
$$

Associated with (3) is a diffusion process with infinitesimal mean $a(p)$ and infinitesimal variance $\sigma^{2}(p)$ (Etheridge 2012; Ewens 2004; Gardiner 2009). In this context, $a(p)$ represents directional biases favouring a particular strain (through either mutation or selection), whereas $\sigma^{2}(p)$ represents non-directional (or unbiased) evolutionary noise due to demographic stochasticity.

As we will see, when selection is sufficiently weak, demographic stochasticity plays a key role in the evolutionary process, even in large populations. This may seem surprising: typically, demographic stochasticity is viewed as a small effect unless population size is also very small (Lande et al. 2003). In our model this is true when we are dealing with processes operating on the fast timescale of (1): in that case, the dynamics are largely driven by the expected change (the terms multiplied by $d t$ ) since the stochastic noise terms are scaled by $\Omega^{-1 / 2}$ (which is small for large population sizes). However, when the system is in the vicinity of the slow manifold, the expected change in population density becomes smaller and smaller (since on the slow manifold $\beta(\mathbf{x})=\mu(\mathbf{x})$ ), whereas this is not true for the stochasticity. Thus if selection is weak and mutations are rare, on the slow timescale the effects of selection and mutation are of comparable magnitude to the effect of demographic stochasticity, and so demographic stochasticity can play a significant role (Fig. 1)

\section{Results}

We wish to use (3) to understand how selection, stochasticity, and epidemiology interact to shape the evolution of sterility virulence. To do so we adopt the approach we used elsewhere 
(McLeod and Day 2019), and ask what is the likelihood of observing the stochastic process in a particular state? If we are more likely to observe the process in a state where strain $i$ is most frequent, then we will say strain $i$ is favoured. In what follows we provide a brief summary of our analysis, with an emphasis on providing intuition for our results; the full analysis can be found in the Supplementary Information (SI 3). Our first objective will be to understand the evolution of sterility virulence when there is no link between sterility virulence and transmission (i.e., $\varepsilon=0$ ), to see how epidemiology alone can impact which strain is favoured. We will then briefly consider the possibility of a link between transmission and sterility virulence to understand how robust our predictions are in the presence of selection.

When there is no link between transmission and sterility virulence $(\varepsilon=0)$, then there are only two factors in (3): (i) unbiased mutations, pushing the population towards $p=1 / 2$, and (ii) the role played by the infinitesimal variance, $\sigma^{2}(p)$, which need not be symmetric and instead will vary in magnitude dependent upon the pathogen population composition. But when the infinitesimal variance is large (resp. small), evolutionary noise will be large (resp. small) causing the system to exhibit more variation. As a consequence, the stochastic process will spend less time in states with large evolutionary noise (large $\sigma^{2}(p)$ ) and so will be less likely to be observed in these states. Hence in the absence of selection, the pathogen strain minimizing $\sigma^{2}(p)$ is stochastically favoured (SI 3). Since the factor $p(1-p) / \Omega$ in $\sigma^{2}(p)$ is symmetric in $p$, the strain minimizing $\sigma^{2}(p)$ will be the strain which minimizes $\mathfrak{R}\left(\mathbf{x}_{p}\right)$ (see also SI 3).

The biological importance of $\mathfrak{R}\left(\mathbf{x}_{p}\right)$ can be easily understood. The numerator of $\mathfrak{R}\left(\mathbf{x}_{p}\right)$, $\beta\left(\mathbf{x}_{p}\right)+\mu\left(\mathbf{x}_{p}\right)$, represents the variance in per capita pathogen growth rate at selective neutrality. As the variance in per capita growth increases, pathogen population turnover also increases, causing the population composition to change more rapidly. Counteracting this force is the effect of total pathogen population density, $\mathcal{I}(p)$ (the denominator of $\mathfrak{R}\left(\mathbf{x}_{p}\right)$ ): the larger the pathogen population (as measured by $\mathcal{I}(p)$ ), the more robust the population is to demographic fluctuations since the net change in population composition due to a strain $i$ infected host being replaced by a strain $j$ infected host will be smaller. These two effects can 
be understood by analogy to a random walk where the walker's position is $p$ : if we increase the variance in per capita growth rate, we decrease time between steps taken by the walker, whereas if we decrease population size, we increase the size of each step. A walker taking larger, more frequent steps will exit a region more rapidly, despite having no directional bias in its movement, and so will be less likely to be observed in such a region. Thus the ratio of the variance in per capita growth rate to total population size can be roughly thought of as a measure for how rapidly the stochastic process moves on the evolutionary timescale. The strain whose sterility virulence 'slows' this process the most will be favoured. This is because if the stochastic process gets 'bogged down' for a particular strain composition and so population composition changes slowly, then we will be more likely to observe this strain composition in the evolving population at any given point in time. With this in mind, we now consider how epidemiology alters the level of sterility virulence which minimizes $\mathfrak{R}\left(\mathbf{x}_{p}\right)$.

\section{Role of epidemiology}

We are interested in how epidemiology affects the level of sterility virulence minimizing $\mathfrak{R}\left(\mathbf{x}_{p}\right)$. To determine this, assume the two pathogen strains are selectively neutral $(\varepsilon=0)$ and are nearly identical in sterility virulence, $\delta_{1}=\delta$ and $\delta_{2}=\delta+\Delta \delta$ with $\Delta \delta \approx 0$. Then, to determine which strain minimizes $\mathfrak{R}\left(\mathbf{x}_{p}\right)$, a first-order Taylor expansion of $\mathfrak{R}\left(\mathbf{x}_{p}\right)$ in $\Delta \delta$ reveals that it is sufficient to consider the sign of $d \mathfrak{R} / d \delta$, evaluated when $\delta_{1}=\delta_{2}=\delta$. In particular, if $d \Re / d \delta>0$, pathogen strains causing less host sterility will be favoured, whereas if $d \Re / d \delta<0$, pathogen strains causing greater host sterility will be favoured. Thus we wish to determine the sign of $d \mathfrak{R} / d \delta$. For this analysis, since neither $\mathcal{I}(p)$ nor $x_{p}$ will depend upon the composition of the pathogen population, $p$, to distinguish this case from the more general case we let $\mathcal{I}(p)=\overline{\mathcal{I}}$ and $x_{p}=\bar{x}$.

To determine the sign of $d \Re / d \delta$, we will focus upon the transmission function $\beta(\mathbf{x})=$ $B x /(x+y+c)$ where $y=\sum_{i} y_{i}$ and $B, c$ are constants. The motivation for choosing this transmission function is that by varying the parameters $B$ and $c$ we can investigate everything from exclusively frequency-dependent transmission $(c=0)$ to exclusively density-dependent 
transmission $(c \rightarrow \infty)$. Note that when we take the limit as $c \rightarrow \infty$ we assume that $B$ increases in proportion to $c$ so that the ratio $B / c$ is a non-zero, finite constant. Using this transmission function, by implicit differentiation (see SI 4) we can compute

$$
\frac{d \mathfrak{R}}{d \delta} \propto\left[-\frac{x+y}{x+y+c} x \frac{\partial \mu}{\partial x}+\frac{y+c}{x+y+c}\left(\mu(x, y)-x \frac{\partial \mu}{\partial x}-y \frac{\partial \mu}{\partial y}\right)\right]_{\substack{x=\bar{x} \\ y=\overline{\mathcal{I}}}},
$$

where proportionality is up to a positive factor. From equation (5), we see that there are two primary factors determining whether sterility virulence is stochastically favoured or disfavoured: (i) the influence of density-dependent mortality (controlled by the derivatives $\frac{\partial \mu}{\partial x}, \frac{\partial \mu}{\partial y}$ ), and (ii) the degree to which transmission is either frequency- or density-dependent (controlled by $c$ ). Clearly, if mortality is unaffected by population density (i.e., $\partial \mu / \partial x=$ $\partial \mu / \partial y=0)$, then $d \mathfrak{R} / d \delta>0$ and so sterility virulence is never stochastically favoured. However, as the strength of density-dependent mortality increases, so too do the potential benefits of sterility virulence. If mortality is positively density-dependent (i.e., $\partial \mu / \partial x>0$, $\partial \mu / \partial y>0)$, then as transmission becomes increasingly frequency-dependent $(c \rightarrow 0)$, the likelihood that $d \mathfrak{R} / d \delta<0$ increases. This can be shown by computing the derivative of (5) with respect to $c$ (while holding all else constant): doing so, we see that if $\mu(x, y)+\frac{\partial \mu}{\partial x} y>\frac{\partial \mu}{\partial y} y$, then (5) is a monotonically increasing function of $c$. The condition $\mu(x, y)+\frac{\partial \mu}{\partial x} y>\frac{\partial \mu}{\partial y} y$ will hold for most, if not all, biologically plausible mortality functions; for example, susceptible hosts being equal or superior competitors to infected hosts is sufficient (e.g., $\mu(\mathbf{x})=a_{0}+$ $a_{1} x+a_{2}\left(y_{1}+y_{2}\right)$ with $\left.a_{1} \geq a_{2}\right)$.

To understand these results, observe that to minimize $\mathfrak{R}\left(\mathbf{x}_{p}\right)$ the pathogen strain needs to reduce its variance in per capita growth (the numerator of $\mathfrak{R}\left(\mathbf{x}_{p}\right)$ ), while simultaneously increasing its equilibrium density (the denominator of $\mathfrak{R}\left(\mathbf{x}_{p}\right)$ ). It can be shown that the variance in per capita growth is a non-increasing function of sterility virulence (SI 4). The intuitive reason for this is that increasing sterility virulence reduces the density of susceptibles, lowering the rate at which transmission and death events occur, thereby decreasing the variance in per capita growth of the pathogen. Because increasing sterility virulence reduces 
the variance in per capita growth (decreasing the numerator of $\mathfrak{R}\left(\mathbf{x}_{p}\right)$ ) and so favouring sterility virulence, the necessary (but not sufficient) condition for sterility to be stochastically disfavoured is that an increase in sterility virulence must also decrease pathogen density (decrease the denominator of $\mathfrak{R}\left(\mathbf{x}_{p}\right)$ ). As sterility virulence only indirectly affects pathogen density through its negative effect on susceptible density, pathogen density is reduced by sterility virulence only if a decrease in susceptible density has a greater impact upon transmission, $\beta(\mathbf{x})$, than on infected host mortality, $\mu(\mathbf{x})$. The likelihood of this will increase as the degree to which transmission is density-dependent increases, and the weaker the dependence of host mortality upon susceptible density, in agreement with our analysis of (5) (Fig. $2 \mathbf{e}-\mathbf{h})$.

The other thing to note is that (5) only indirectly depends upon the birth and death rates of the susceptible population. As a result, the birth and death rates of susceptibles can assume any form satisfying the assumptions leading to model (1) and the results from consideration of (5) will not be altered. However, in order for sterility virulence to be favoured by frequency-dependent transmission, the 'strength' of density-dependent mortality cannot be too weak. In some populations, population size will be primarily regulated by densitydependent fecundity and only weakly impacted by density-dependent mortality. In this circumstance it can be shown that if the basic reproductive ratio of the pathogen approaches unity, sterility virulence is favoured for frequency-dependent transmission (SI 4).

To provide a concrete example of when sterility virulence is favoured or disfavoured, consider the model

$$
\begin{aligned}
& b(\mathbf{x})=\tilde{b}\left(x+\sum_{i}\left[1-\delta_{i}\right] y_{i}\right) \\
& d(\mathbf{x})=m+\kappa\left(x+\sum_{i} y_{i}\right) \\
& \mu(\mathbf{x})=\alpha+d(\mathbf{x})
\end{aligned}
$$

where $\tilde{b}$ is the per capita rate of reproduction, $m$ is natural mortality, $\alpha$ is virulence-related mortality, and $\kappa$ controls the magnitude of density-dependent mortality. Set $y=\sum_{i} y_{i}$ and suppose that $\delta_{i}=\delta$ for all $i$, so that all strains have equal sterility virulence. Then from (5) if transmission is exclusively density-dependent, $\beta(\mathbf{x})=B x$ (so $c \rightarrow \infty$ in (5)), this 
leaves (5) equal in the limit of $c \rightarrow \infty$ to $\alpha+m$. Hence for density-dependent transmission, pathogens causing no sterility are favoured. Conversely, if transmission is exclusively frequency-dependent, $\beta(\mathbf{x})=B x /(x+y)$, the level of sterility minimizing $\mathfrak{R}\left(\mathbf{x}_{p}\right)$ can be computed directly by solving $[d \mathfrak{R} / d \delta]_{\delta=\delta^{*}}=0$ for $\delta^{*}$ : as the quantities are evaluated for $\delta_{1}=\delta_{2}=\delta$, on the slow timescale this will not depend upon $p$ (nor will $\mathfrak{R}\left(\mathbf{x}_{p}\right)$ ). Doing so yields

$$
\delta^{*}=1-\frac{(B-\alpha-\tilde{b})(m+\alpha+\sqrt{B(m+\alpha)})}{\tilde{b}(B-m-\alpha)},
$$

and so pathogens causing some level of sterility are stochastically favoured, and the optimal level of sterility is $\delta^{*}$.

Although our analytic predictions have focused upon the two-strain case, we can extend (1) to include $N$ strains, each with a different sterility virulence, such that

$$
\begin{aligned}
d x & =\left[b(\mathbf{x})-d(\mathbf{x}) x-\sum_{i} \beta(\mathbf{x}) y_{i}\right] d t+\Omega^{-1 / 2} M_{x} \\
d y_{i} & =\left[(\beta(\mathbf{x})-\mu(\mathbf{x})) y_{i}+\nu\left(\sum_{j} y_{j}-N y_{i}\right)\right] d t+\Omega^{-1 / 2} M_{y_{i}}, \quad i=1,2, \ldots, N
\end{aligned}
$$

where $y_{i}$ is the density of the $i$-th strain, $\mathbf{x}=\left(x, y_{1}, y_{2}, \ldots, y_{N}\right)$, and $M_{x}$ and $M_{y_{i}}$ are stochastic unbiased noise terms (see SI 5 for full details). Note that in equation (8) we have assumed that strain $i$ mutates to strain $j$ at a per capita rate $\nu$, and so the total per capita rate at which strain $i$ will mutate to a different strain is $\nu(N-1)$, however, if we were to instead use a single-step mutational scheme it will not qualitatively change our results (see SI 5). In Figure 2 we simulate system (8) with $N=7$ using the Euler-Maruyama method (Allen 2011; see also SI 5), where we have used the model specified by equations (6) with $\beta(\mathbf{x})=\frac{B x}{1+q\left(x+\sum_{i} y_{i}\right)}$. Hence as we decrease $q$ from subplot a to subplot $\mathbf{d}$, transmission becomes increasingly density-dependent. The simulation results show that the predictions of our two-strain model scale as expected when there are more strains present in the population. In particular, when transmission is predominantly frequency-dependent (Fig. 2a), fully sterilizing pathogens are favoured, whereas when transmission is increasingly density-dependent (Fig. 2d), sterility 
virulence becomes disfavoured, in agreement with our general predictions obtained from consideration of (5). Additionally, the stochastically favoured level of sterility virulence, $\delta^{*}$, for the $N$ strain model matches well with the level of virulence minimizing $\mathfrak{R}\left(\mathbf{x}_{p}\right)$ obtained in the two-strain case.

Now, when the population becomes infinitely large, $\Omega \rightarrow \infty$, demographic stochasticity is negligible, and so each pathogen phenotype is equally likely to be observed in the population, irrespective of its sterility virulence. Thus we may ask how small populations have to be for demographic stochasticity to have an appreciable effect. In Figure 3, we simulate the multi-strain model (8) as presented in Figure 2, for a range of population sizes (by varying $\Omega$ ), revealing that even in large populations demographic stochasticity can play the role predicted by our analysis of the two-strain model. Note as well that the impact of demographic stochasticity inversely scales with the magnitude of directional biases (the strength of selection and the frequency of mutations). If there is a strong link between sterility virulence and transmission (so selection is strong), or mutations are too frequent, demographic stochasticity will play a limited role. This can be seen more explicitly by considering (3): since $d W_{t}$ is a Gaussian random variable with mean zero and variance $d t, d W_{t}=\mathcal{O}(\sqrt{d t})$. Therefore if selection or mutation is strong (i.e., $\varepsilon \gg 1 / \Omega$ or $\nu \gg 1 / \Omega$ ), the influence of the directional biases in (3) (as measured by $a(p)$ ) will greatly exceed the influence of stochasticity (as measured by $\left.\sigma^{2}(p)\right)$.

\section{What happens when transmissibility and sterility virulence are}

\section{linked?}

Thus far we have assumed there is no link between sterility virulence and transmissibility, that is, sterility virulence is selectively neutral. This was purposeful: (i) the evidence for a transmission-sterility virulence trade-off is limited (Abbate et al. 2015), and (ii) we wanted to understand the effect of demographic stochasticity upon the likelihood of observing different pathogen phenotypes in the population. The results of the previous section showed that demographic stochasticity tends to favour sterility virulence as transmission becomes 
increasingly frequency-dependent, provided mortality is also density-dependent. However, we may ask how robust this conclusion is if, as previous studies have considered (Jaenike 1996; O'Keefe 2005; O'Keefe and Antonovics 2002), there is a positive link between sterilityvirulence and transmission. Because in well-mixed populations susceptible hosts represent a public good freely available to all competing pathogen strains, the deterministic model $(\Omega \rightarrow \infty)$ predicts pathogens should sterilize (Jaenike 1996; O'Keefe 2005; O'Keefe and Antonovics 2002). This result holds irrespective of whether transmission is predominantly frequency- or density-dependent.

If the strain at the selective disadvantage also minimizes $\mathfrak{R}\left(\mathbf{x}_{p}\right)$, however, then demographic stochasticity will act in opposition to selection. This occurs if transmission is predominantly density-dependent, or mortality is independent of population density. In this circumstance, we may ask how weak does selection have to be relative to the effect of demographic stochasticity. This can be thought of as asking how small the between-strain difference in transmissibility has to be relative to the between-strain difference in sterility virulence. Our purpose here is not to consider different possible trade-off functions, or to construct a stochastic analogue of adaptive dynamics. Rather, our objective is to consider how robust the effects of demographic stochasticity are relative to selection, when they act in opposition.

To do so, we suppose strain 2 has the transmission advantage, $\beta_{1}(\mathbf{x})=\beta(\mathbf{x})(1-\varepsilon)$, $\beta_{2}(\mathbf{x})=\beta(\mathbf{x})$, and causes increased sterility virulence, such that $\delta_{2}=\delta$ and $\delta_{1}=\delta-\omega \varepsilon$ with $\omega \varepsilon \in[0, \delta]$. Here, $\omega$ is a positive scaling factor (so $\delta_{1}<\delta_{2}$ ) linking between strain differences in transmissibility to between strain differences in sterility (i.e., if $\omega=1$, then a one unit change in between-strain transmissibility corresponds to a one unit change in between-strain sterility). We provide a more comprehensive analysis in the Supplementary Information (SI 3 ), but here we focus only upon the case in which mutations are very rare as the qualitative patterns are similar for larger mutation rates. In this regime, assuming selection is weak, 
the strain with lower transmission and lower sterility virulence (strain 1) is favoured if

$$
\left.\frac{\omega}{2 \Omega} \frac{\partial \mathfrak{R}}{\partial \delta}\right|_{\varepsilon=0}>\beta\left(x_{0}\right)
$$

where $x_{0}$ is the density of susceptibles when $\varepsilon=0$. If the value of $\omega / \Omega$ required to satisfy (9) decreases, either by reducing population size (by decreasing habitat size, $\Omega$ ) or through increasing the between-strain difference in sterility virulence relative to the between-strain difference in transmissibility (by increasing $\omega$ ), then there is a corresponding decline in the likelihood of the evolution of more transmissible pathogens (with higher sterility virulence). Note that the critical value of $\omega / \Omega$ required to satisfy (9) can be related to the strength of selection by observing that by definition of $\omega$, we must have $\omega \leq \delta / \varepsilon$. This in turn implies that $\omega / \Omega \leq \delta /[\varepsilon \Omega]$, and so if, for example, $\varepsilon=1 / \Omega$, then a stochastic reversal would require both (9) to be satisfied, and $\omega / \Omega<\delta$. Thus the between-strain difference in transmissibility (the selective advantage of sterility virulence) must be small in order for the effects of selection to be overturned by demographic stochasticity.

The biological interpretation of (9) is clear: the right-hand side (RHS) is the (selective) transmission costs paid by the less sterilizing strain (the between-strain difference in transmissibility is $\varepsilon \beta\left(x_{0}\right)$, but as everything in (9) is first-order in $\varepsilon, \varepsilon$ cancels from both sides; SI 3), while the left-hand side (LHS) are any non-selective benefits of reduced sterility. To satisfy (9), at minimum the strain with reduced sterility virulence must minimize the ratio $\mathfrak{R}\left(\mathbf{x}_{p}\right)$, that is $\frac{\partial \mathfrak{R}}{\partial \delta}>0$. Hence we can conceptually view inequality (9) as asking when a strain paying a directional bias cost (RHS of (9)) can be stochastically favoured by "slowing" the stochastic process through minimizing $\mathfrak{R}\left(\mathbf{x}_{p}\right)$ (LHS of $(9)$ ).

To gain some more insight into (9) and what favours or disfavours a reversal of selection, focus again upon the model specified by (6) with density-dependent transmission, $\beta(\mathbf{x})=$ $B x$. Our previous analysis showed that for this model, non-sterilizing pathogens minimize $\mathfrak{R}\left(\mathbf{x}_{p}\right)$. Treating inequality (9) as an equality and solving for the critical ratio $\omega / \Omega$, it can be shown that as the basic reproductive ratio, $R_{0}=B(\tilde{b}-m) /(\kappa[\alpha+\tilde{b}])$, of the (neutral) 
pathogen strain becomes small $\left(R_{0} \rightarrow 1\right)$, sterilizing pathogens are increasingly likely to be disfavoured (Fig. 4d-f). This could occur by increasing mortality (increasing $\alpha, m$, or $\kappa$; Fig. $4 \mathbf{b}, \mathbf{c}$ ) or by decreasing transmissibility (decreasing $B$; Fig. $4 \mathbf{a}$ ). On the other hand, if $R_{0}$ becomes large, then a variety of outcomes are possible, depending upon which demographic quantity is manipulated to increase $R_{0}$. If we increase transmissibility, then for large (and small) $R_{0}$, sterilizing pathogens are increasingly likely to be disfavoured (Fig. $4 \mathbf{d}$ ). Conversely, if we decrease natural- and virulence-related mortality, then whether reductions in mortality (and so increases in $R_{0}$ ) cause sterilizing pathogens to be increasingly favoured or disfavoured depends upon a complex interaction with the other parameters (Fig. 4e,f). That these predictions depend upon what assumptions are made about the underlying population demographics emphasizes that not all forms of density-dependence are equivalent, and that how density-dependence is modelled can lead to very different conclusions (Metz et al. 2008; Mylius and Diekmann 1995).

Finally, we note that although we assumed a positive link between sterility virulence and transmission in keeping with previous work (Jaenike 1996; O'Keefe 2005; O'Keefe and Antonovics 2002), if instead there was a negative link beween sterility virulence and transmission, then whenever $\partial \Re / \partial \delta>0$, selection and demographic stochasticity work together to disfavour sterility virulence. Conversely, if $\partial \mathfrak{R} / \partial \delta<0$, then selection and demographic stochasticity work in opposition. In this situation, whenever $-\beta\left(x_{0}\right)<\left.\frac{\omega}{2 \Omega} \frac{\partial \Re}{\partial \delta}\right|_{\varepsilon=0}$ the influence of demographic stochasticity can reverse selection, leading to sterility virulence being stochastically favoured. This can be shown by slightly modifying the arguments used to derive (9).

\section{Discussion}

The fact that sterility virulence is common for STIs but rare for other directly-transmitted pathogens, while other forms of virulence tend to be limited for STIs (Antonovics et al. 2011; Lockhart et al. 1996), has led to the suggestion that sterility virulence may have an adaptive 


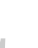

explanation and be 'targeted' (Apari et al. 2014). In particular, it has been hypothesized that by sterilizing, STIs may promote host sexual activity (Apari et al. 2014), and so adaptively increase STI transmission. However, this hypothesis requires a number of assumptions (e.g., pair bonds, ability to divorce and remate) that are not commonly associated with host-STI systems, limiting its explanatory power.

One epidemiological difference between STIs and directly-transmitted pathogens is the form of transmission: STIs tend to exhibit frequency-dependent transmission, whereas directlytransmitted pathogens tend to exhibit mainly density-dependent transmission (e.g., Antonovics et al. 2011). This is because the number of sexual partners an individual has usually depends upon mating system and is only weakly affected by population density (McCallum et al. 2001), and in many sexually reproducing species, mating rate is limited by breeding season, pair-formation, and gestation period and not population density (Lockhart et al. 1996). Indeed, the relationship between frequency-dependent transmission and sexuallytransmitted infections can be derived from first principles (Lloyd-Smith et al. 2004), but is also supported by models fit to data (Anderson and May 1991; Augustine 1998; May and Anderson 1987; Ryder et al. 2014; Webberley et al. 2006). Here we have shown that sterility virulence may indeed be 'targeted' to STIs as argued elsewhere (Apari et al. 2014), but that such a relationship can be explained by consideration of the transmission differences between STIs and directly-transmitted pathogens alone. In particular, provided there is some density-dependent mortality, frequency-dependent transmission tends to favour sterility virulence whereas density-dependent transmission disfavours sterility virulence. Our results can be applied to more complex combinations of frequency- and density-dependent transmission which sometimes better fit empirical data (e.g., McCallum et al. 2017; Ryder et al. 2005; Smith et al. 2009) such that 'mainly' frequency-dependent transmission favours sterility virulence whereas 'mainly' density-dependent transmission disfavours sterility (Fig. 2). Moreover, we have shown that even if there is a (weak) selective advantage to sterilizing in terms of increased transmissibility, stochasticity can be sufficient to overcome selection (Fig. 4), and that this is most likely to occur for pathogens with either low $R_{0}$ or high 
transmissibility.

Although our prediction that stochasticity favours sterilizing STIs requires some form of density-dependent mortality of infected individuals, we suggest that this is likely not an overly stringent assumption. Not only are STIs commonly associated with low levels of virulence (other than sterility; Antonovics et al. 2011; Apari et al. 2014; Lockhart et al. 1996), they are often chronic (Antonovics et al. 2011) and so we should not expect being infected to shield or remove individuals from intraspecific competition that would otherwise occur. As such, it seems reasonable to expect that infected hosts will be subject to the same demographic forces as susceptible hosts, and many populations are known to be subject to density-dependent adult mortality, through for example predation (e.g., lemmings; Gilg et al. 2003), or intraspecific competition and aggression over territories (e.g., wolves; Cubaynes et al. 2014) and resources (e.g., red deer and birds; Clutton-Brock et al. 2002; Saether et al. 2016).

Examining the role played by different demographic assumptions has a long history in life history modeling, with the focus typically upon when evolution admits an optimization principle (Metz et al. 2008; Mylius and Diekmann 1995). Applications to host-pathogen systems have considered deterministic models, and so the primary interest is when demographic feedbacks can create the potential for adaptive diversification and evolutionary branching (e.g., Best et al. 2009; Boldin and Kisdi 2012; Cortez 2013; Pugliese 2002), rather than the interplay between population demography and stochasticity, as we have considered here. Our work thus suggests an additional implication of demographic assumptions for host-pathogen evolution when finite population sizes are considered; moreover, the framework we have employed here may be used to investigate other forms of less commonly considered pathogen virulence (such as morbidity) that often have limited evolutionary implications in standard, deterministic host-pathogen models.

Existing theory has tended to focus upon determining what factors select against sterility virulence without explicitly addressing why sterility virulence is more commonly associated with STIs than directly-transmitted pathogens. However, a central assumption of existing 
models is that population size is large (ideally infinite) and so stochasticity is ignored. As a result, epidemiological differences between STIs and other directly-transmitted pathogens, such as the form of transmission function, tend to play a limited evolutionary role. By focusing upon finite population sizes, here we have shown the importance of epidemiology upon the evolution of sterility virulence. 


\section{References}

Abbate, J. L., S. Kada, and S. Lion. 2015. Beyond mortality: sterility as a neglected component of parasite virulence. PLoS Pathog. 11.

Allen, L. J. S., 2011. An Introduction to Stochastic Processes with Applications to Biology. CRC Press, Boca Raton, FL.

Anderson, R. M. and R. M. May. 1979. Population biology of infectious diseases: Part I. Nature 280:361-367.

, 1991. Infectious diseases of humans: dynamics and control. Oxford University Press, Oxford, UK.

Antonovics, J., M. Boots, J. Abbate, C. Baker, Q. McFrederick, and V. Panjeti. 2011. Biology and evolution of sexual transmission. Ann. N.Y. Acad. Sci. Pp. 12-24.

Apari, P., J. D. de Sousa, and V. Muller. 2014. Why sexually transmitted infections tend to cause infertility: an evolutionary hypothesis. PLoS Pathog 10:e1004111.

Ashby, B. and M. Boots. 2015. Coevolution of parasite virulence and host mating strategies. Proc. Natl. Acad. Sci. 112:13290-13295.

Augustine, D. J. 1998. Modelling chlamydia-koala interactions: coexistence, population dynamics and conservation implications. J. Appl. Ecol. 35:261-272.

Begon, M., M. Bennett, R. G. Bowers, N. P. French, S. M. Hazel, and J. Turner. 2002. A clarification of transmission terms in host-microparasite models: numbers, densities and areas. Epidemiol. Infect. 129:147-153.

Berglund, N. and B. Gentz, 2006. Noise-induced Phenomena in Slow-Fast Dynamical Systems. Springer, USA.

Best, A., A. White, and M. Boots. 2009. The implications of coevolutionary dynamics to host-parasite interactions. Am. Nat. 173:779-791.

2010. Resistance is futile but tolerance can explain why parasites do not always castrate their hosts. Evolution 64:348-357.

Boldin, B. and E. Kisdi. 2012. On the evolutionary dynamics of pathogens with direct and environmental transmission. Evolution 66:2514-2527.

Clutton-Brock, T. H., T. N. Coulson, E. J. Milner-Gulland, D. Thomson, and H. M. Armstrong. 2002. Sex differences in emigration and mortality affect optimal management of deer populations. Nature 415:633-637.

Constable, G. W. A., T. Rogers, A. J. McKane, and C. E. Tarnita. 2016. Demographic noise can reverse the direction of deterministic selection. Proc. Natl. Acad. Sci. .

Cortez, M. H. 2013. When does pathogen evolution maximize the basic reproductive number in well-mixed host-pathogen systems? J. Math. Biol. 67:1533-1585. 

$\bigcup^{2}$ 506

Cubaynes, S., D. R. MacNulty, D. R. Stahler, K. A. Quimby, D. W. Smith, and T. Coulson. 2014. Density-dependent intraspecific aggression regulates survival in northern Yellowstone wolves (Canis lupus). J. Anim. Ecol. 83:1344-1356.

de Castro, F. and B. Bolker. 2005. Mechanisms of disease-induced extinction. Ecol. Lett. 8:117-126.

Etheridge, A., 2012. Some mathematical models from population genetics. Springer-Verlag, New York, NY.

Ewens, W. J., 2004. Mathematical Population Genetics I: Theoretical Introduction. Springer: Interdisciplinary Applied Mathematics, New York, NY.

Gardiner, C. W., 2009. Handbook of Stochastic Methods. Springer: Complexity, Berlin, Germany.

Getz, W. M. and J. Pickering. 1983. Thresholds and population regulation. Am. Nat. 121:892-898.

Gilg, O., I. Hanski, and B. Sittler. 2003. Cyclic dynamics in a simple vertebrate predator-prey community. Science 302:866-868.

Gillespie, D. T. 1977. Exact stochastic simulation of coupled chemical reactions. J. Phys. Chem. 81:2340-2361.

Hurst, G. D. D., R. G. Sharpe, A. H. Broomfield, L. E. Walker, T. M. O. Majerus, I. A. Zakharov, and M. E. N. Majerus. 1995. Sexually transmitted disease in a promiscuous insect, Adalia bipunctata. Ecol. Entomol. 20:230-236.

Jaenike, J. 1996. Suboptimal virulence of an insect-parasitic nematode. Evolution 50:22412247.

Karlin, S. and H. M. Taylor, 1981. A Second Course in Stochastic Processes. Academic Press, New York, NY.

Katzenberg, G. S. 1991. Solutions of a stochastic differential equation forced onto a manifold by a large drift. Ann. Probab. 19:1587-1628.

Kimura, M. 1968. Evolutionary rate at the molecular level. Nature 217:624-626.

King, J. L. and T. H. Jukes. 1969. Non-Darwinian evolution. Science 164:788-797.

Knell, R. J. and K. M. Webberley. 2004. Sexually transmitted diseases of insects: distribution, evolution, ecology and host behaviour. Biol. Rev. 79:557-581.

Lande, R., S. Engen, and B. Saether, 2003. Stochastic Population Dynamics in Ecology and Conservation. Oxford University Press, Oxford, UK.

Lion, S. and S. Gandon. 2015. Evolution of spatially structured host-parasite interactions. J. Evol. Biol. 28:10-28. 
Lloyd-Smith, J. O., W. M. Getz, and H. V. Westerhoff. 2004. Frequency-dependent incidence in models of sexually transmitted diseases: portrayal of pair-based transmission and effects of illness on contact behaviour. Proc. R. Soc. B 271:625-635.

Lockhart, A. B., P. H. Thrall, and J. Antonovics. 1996. Sexually transmitted diseases in animals: ecological and evolutionary implications. Biol. Rev. 71:415-471.

May, R. M. and R. M. Anderson. 1987. Transmission dynamics of HIV infection. Nature $326: 137-142$.

McCallum, H., N. Barlow, and J. Hone. 2001. How should pathogen transmission be modelled? Trends Ecol. Evol. 16:295-300.

McCallum, H., A. Fenton, P. J. Hudson, B. Lee, B. Levick, R. Normal, S. E. Perkins, M. Viney, A. J. Wilson, and J. Lello. 2017. Breaking beta: deconstructing the parasite transmission function. Phil. Trans. R. Soc. B 372:20160084.

McCandlish, D. M. and A. Stoltzfus. 2014. Modeling evolution using the probability of fixation: history and implications. Quarterly Review of Biology 89:225-252.

McLeod, D. V. and T. Day. 2015. Pathogen evolution under host avoidance plasticity. Proc. R. Soc. B 282:20151656.

. 2019. Social evolution under demographic stochasticity. PLoS Comp. Biol. 15:1-13. https://doi.org/10.1371/journal.pcbi.1006739.

Metz, J. A. J., S. D. Mylius, and O. Diekmann. 2008. When does evolution optimize? Evol. Ecol. Res. 10:629-654.

Mylius, S. D. and O. Diekmann. 1995. On evolutionarily stable life histories, optimization and the need to be specific about density dependence. Oikos 74:218-224.

O'Keefe, K. J. 2005. The evolution of virulence in pathogens with frequency-dependent transmission. J. Theor. Biol. 233:55-64.

O'Keefe, K. J. and J. Antonovics. 2002. Playing by different rules: the evolution of virulence in sterilizing pathogens. Am. Nat. 159:597-605.

Parsons, T. L. and T. Rogers. 2017. Dimension reduction for stochastic dynamical systems forced onto a manifold by large drift: a constructive approach with examples from theoretical biology. J. Phys. A: Math. Theor. 50.

Pugliese, A. 2002. On the evolutionary coexistence of parasite strains. Mathematical Biosciences 177:355-375.

Ryder, J., M. Hoare, D. Pastok, M. Bottery, M. Boots, A. Fenton, D. Atkinson, R. J. Knell, and G. D. D. Hurst. 2014. Disease epidemiology in arthropods is altered by the presence of nonprotective symbionts. Am. Nat. 183:E89-E104.

Ryder, J. J., K. M. Webberley, M. Boots, and R. J. Knell. 2005. Measuring the transmission dynamics of a sexually transmitted disease. Proc. Natl. Acad. Sci. 102:15140-15143. 
Saether, B., G. V., S. Engen, T. Coulson, P. R. Grant, M. E. Visser, J. E. Brommer, B. R. Grant, L. Gustafsson, B. J. Hatchwell, K. Jerstad, P. Karell, H. Pietiainen, A. Roulin, O. W. Rostad, and H. Weimerskirch. 2016. Demographic routes to variability and regulation in bird populations. Nat. Comm. 7:12001.

Smith, G. and A. P. Dobson. 1992. Sexually transmitted diseases in animals. Parasitology Today 8:159-166.

Smith, M., S. Telfer, E. R. Kallio, S. Burthe, A. R. Cook, X. Lambin, and M. Begon. 2009. Host-pathogen time series data in wildlife support a transmission function between density and frequency dependence. Proc. Natl. Acad. Sci. 106:7905-7909.

van Kampen, N. G., 1981. Stochastic Processes in Physics and Chemistry. North-Holland, New York, NY.

Webberley, K. M., J. Buszko, V. Isham, and G. D. D. Hurst. 2006. Sexually transmitted disease epidemics in a natural insect population. J. Anim. Ecol. 75:33-43.

Weigler, B. J., A. A. Girjes, N. A. White, N. D. Kunst, F. N. Carrick, and M. F. Lavin. 1988. Aspects of the epidemiology of Chlamydia psittaci infection in a population of koalas (Phascolarctos cinereus) in southeastern Queensland, Australia. J. Wildlife Diseases 24:282-291. 
Figure 1: Illustration of the fast- and slow-timescales associated with system (1). Subplots a and $\mathbf{b}$ show sample trajectories of the deterministic dynamics (smooth curves) and stochastic dynamics (rough curves) of the full stochastic process approximated by (1); time has been rescaled so that both the fast- and slow-timescales are visible. Initially, the expected change in (1) is the dominant force and so there is a close match between the deterministic and stochastic dynamics (fast-timescale). However, once the system has moved into the vicinity of the slow manifold, demographic stochasticity comes into play and the stochastic trajectories diverge from the deterministic trajectories. Subplots $\mathbf{g}, \mathbf{h}$ show these dynamics in $\left(x, y_{1}, y_{2}\right)$ space, while subplots c-f show the view from the $\left(y_{1}, y_{2}\right)$-plane. In subplots $\mathbf{c}-\mathbf{h}$ the red curve is the slow manifold, the black curves are deterministic trajectories, and the rough curves are stochastic trajectories. Subplots $\mathbf{a}, \mathbf{c}, \mathbf{d}, \mathbf{g}$ assume there is no selection, while subplots $\mathbf{b}, \mathbf{e}, \mathbf{f}, \mathbf{g}$ assume weak selection favouring strain $2(\varepsilon=0.005)$. For these examples, $\beta_{1}(\mathbf{x})=8 x(1-\varepsilon)$, $\beta_{2}(\mathbf{x})=8 x, b(\mathbf{x})=5\left(x+y_{1}+0.25 y_{2}\right), d(\mathbf{x})=0.25+0.5\left(x+y_{1}+y_{2}\right), \mu(\mathbf{x})=0.25+d(\mathbf{x})$, with $\Omega=10^{3}$ and no mutation $(\nu=0)$. 
Figure 2: The favoured level of sterility virulence depends upon the epidemiology of transmission. As pathogen transmission transitions from mainly frequency-dependent (subplot $\mathbf{a}, \mathbf{e})$ to mainly density-dependent (subplot $\mathbf{d , h}$ ), the favoured sterility virulence phenotype transitions from one causing complete sterility to one causing no sterility in agreement with analytic predictions (the level of sterility virulence which minimizes $\mathfrak{R}\left(\mathbf{x}_{p}\right)$ is the dashed vertical line in subplots a-d). The blue circles in subplots a-d are the stationary distribution obtained from $2.5 \times 10^{4}$ runs of the multistrain model with $N=7$ (see system (8)) simulated using Euler-Maruyama method (see SI 5). Subplots e-h show the expected number of susceptible and infected hosts for the sterility virulence indicated by the $x$-axis. Each column corresponds to a different value of $q$ : from subplot a to $\mathbf{d}, q$ was taken to be $3.9216,3.125,1.9048,0.5$, respectively. The choice of $q$ was made to show the range of potential optimal sterility virulence phenotypes. Parameter values used were $\{B, \alpha, m, \kappa, \tilde{b}, \nu, \Omega\}=\left\{16.8,0.05,0.75,0.3,4,10^{-6}, 10^{4}\right\}$. 
Figure 3: The relationship between population size (controlled by habitat size, $\Omega$ ) and the strength of demographic stochasticity. In subplot $\mathbf{a}$, transmission is mainly frequencydependent, whereas in subplot $\mathbf{b}$, transmission is mainly density-dependent. The circles are the stationary distribution for three different values of $\Omega$, obtained from $2.5 \times 10^{4}$ runs of model (8) with $N=7$ using Euler-Maruyama method. Parameter values used were $\{B, \alpha, m, \kappa, \tilde{b}, \nu, \Omega\}=\left\{16.8,0.05,0.75,0.3,4,10^{-6}, 10^{4}\right\}$, with $q=3.9216$ for subplot a and $q=0.5$ for subplot $\mathbf{b}$ 
Figure 4: Example behaviour of critical ratio $\omega / \Omega$ (subplots $\mathbf{d}-\mathbf{f}$ ) and basic reproductive number, $R_{0}$ (subplots a-c), as demographic parameters, $B, \alpha$, and $m$ are varied. In subplots d-f, for values of $\omega / \Omega$ above (resp. below) the various curves, the strain with decreased transmission and decreased sterility virulence is favoured (resp. disfavoured). Thus when the critical ratio $\omega / \Omega$ is small, strains causing no sterility virulence are increasingly stochastically favoured, despite being selected against. No matter what demographic quantity is varied ( $B, \alpha, m)$, as $R_{0} \rightarrow 1$ (dashed vertical lines correspond to $R_{0}=1$, subplots a-c), the value of $\omega / \Omega$ required to favour reduced sterility virulence also decreases. For this model, the density of susceptibles is an increasing function of $\alpha$ and $m$ and a decreasing function of $B$, and so as $R_{0} \rightarrow 1$, the density of susceptibles becomes maximal. 


\section{Supplementary Information}

\section{Model derivation}

Here we derive the set of stochastic differential equations (SDEs) presented in the main text (equations (1)) from a continuous-time Markov chain with discrete state space. Our focus is a susceptible-infected host-pathogen population with horizontal transmission, no multiple infection and no recovery. Let $X(t)$ and $Y_{i}(t)$ be the number of susceptible hosts and hosts infected with pathogen strain $i=1,2$ at time $t$. Let $\mathbb{X}=\left(X, Y_{1}, Y_{2}\right)$, and suppose $\Omega$ is the system-size parameter controlling intraspecific interactions (e.g., Gardiner 2009; van Kampen 1981). In our context $\Omega$ can be thought of as the habitat size. Let the possible events and transition probabilities per unit time be

$\begin{array}{lll}\text { Event type } & \text { Transition } & \text { Probability per unit time } \\ \text { birth of susceptible } & \mathbb{X} \rightarrow \mathbb{X}+\vec{e}_{1} & T_{b}(\mathbb{X})=b(\mathbb{X}) \\ \text { death of susceptible } & \mathbb{X} \rightarrow \mathbb{X}-\vec{e}_{1} & T_{d}(\mathbb{X})=d(\mathbb{X}) X \\ \text { transmission event } & \mathbb{X} \rightarrow \mathbb{X}-\vec{e}_{1}+\vec{e}_{i+1} & T_{\beta_{i}}(\mathbb{X})=\beta_{i}(\mathbb{X}) Y_{i}, \quad i=1,2 \\ \text { death of infected } & \mathbb{X} \rightarrow \mathbb{X}-\vec{e}_{i+1} & T_{\mu_{i}}(\mathbb{X})=\mu(\mathbb{X}) Y_{i}, \quad i=1,2 \\ \text { pathogen mutation } & \mathbb{X} \rightarrow \mathbb{X}-\vec{e}_{i+1}+\vec{e}_{j+1} & T_{\nu_{i}}(\mathbb{X})=\nu Y_{i}, \quad i, j=1,2, i \neq j\end{array}$

where $\vec{e}_{i}$ is the $3 \times 1$ zero vector with a 1 in the $i$-th spot. Note that all interactions between individuals are scaled by habitat size. For example, if transmission is purely densitydependent, then $\beta(\mathbb{X})=\beta X / \Omega$, while if birth rate is density-dependent, we may have $b(\mathbb{X})=$ $b\left(X+\sum_{i}\left(1-\delta_{i}\right) Y_{i}\right)\left(1-\left[X+Y_{1}+Y_{2}\right] / \Omega\right)$. Let $Q(\mathbb{X}, t)$ be the probability the system is in state $\mathbb{X}$ at time $t$. Then the Master equation for (10) is

$$
\begin{aligned}
& \frac{\partial Q}{\partial t}= T_{b}\left(\mathbb{X}-\vec{e}_{1}\right) Q\left(\mathbb{X}-\vec{e}_{1}, t\right)+T_{d}\left(\mathbb{X}+\vec{e}_{1}\right) Q\left(\mathbb{X}+\vec{e}_{1}, t\right) \\
&+ \sum_{i=1,2}\left[T_{\beta_{i}}\left(\mathbb{X}+\vec{e}_{1}-\vec{e}_{i+1}\right) Q\left(\mathbb{X}+\vec{e}_{1}-\vec{e}_{i+1}, t\right)+T_{\mu_{i}}\left(\mathbb{X}+\vec{e}_{i+1}\right) Q\left(\mathbb{X}+\vec{e}_{i+1}, t\right)\right] \\
&+T_{\nu_{1}}\left(\mathbb{X}+\vec{e}_{2}-\vec{e}_{3}\right) Q\left(\mathbb{X}+\vec{e}_{2}-\vec{e}_{3}, t\right)+T_{\nu_{2}}\left(\mathbb{X}+\vec{e}_{3}-\vec{e}_{2}\right) Q\left(\mathbb{X}+\vec{e}_{3}-\vec{e}_{2}, t\right) \\
& \quad-\left(T_{b}(\mathbb{X})+T_{d}(\mathbb{X})+\sum_{i=1,2}\left[T_{\beta_{i}}(\mathbb{X})+T_{\mu_{i}}(\mathbb{X})+T_{\nu_{i}}(\mathbb{X})\right]\right) Q(\mathbb{X}, t) .
\end{aligned}
$$

Equation (11) describes the time evolution of $Q(\mathbb{X}, t)$, but is analytically intractable. Let $\mathbf{x}=\left(x, y_{1}, y_{2}\right)=\mathbb{X} / \Omega$ and suppose $\Omega$ is sufficiently large such that the variables $\mathbf{x}$ are approximately continuous. Define the new probability density function $q(\mathbf{x}, t) \equiv \Omega Q(\mathbb{X}, t)$, and rescale time as $\tau=t / \Omega$. Then we can expand (11) in powers of $1 / \Omega$, neglecting terms of order $\Omega^{-2}$ (Gardiner 2009; van Kampen 1981), to obtain the Fokker-Planck (or forward Kolmogorov) equation

$$
\frac{\partial q}{\partial \tau}=-\sum_{i=1}^{3} \frac{\partial}{\partial \mathbf{x}_{i}} \mathrm{~A}_{i}(\mathbf{x}) q(\mathbf{x}, \tau)+\frac{1}{2 \Omega} \sum_{i=1}^{3} \sum_{j=1}^{3} \frac{\partial^{2}}{\partial \mathbf{x}_{i} \partial \mathbf{x}_{j}} \mathrm{~B}_{i j}(\mathbf{x}) q(\mathbf{x}, \tau)
$$


where

$$
\begin{aligned}
\mathrm{A}_{1}(\mathbf{x}) & =b(\mathbf{x})-d(\mathbf{x}) x-\sum_{j} \beta_{j}(\mathbf{x}) y_{j}, \\
\mathrm{~A}_{i+1}(\mathbf{x}) & =\left(\beta_{i}(\mathbf{x})-\mu(\mathbf{x})\right) y_{i}+\nu\left(y_{j}-y_{i}\right), i, j=1,2, \quad i \neq j
\end{aligned}
$$

and $\mathrm{B}_{i j}(\mathbf{x})$ is the $i j$-th entry of the matrix

$$
\mathbf{B}(\mathbf{x})=\left(\begin{array}{ccc}
b(\mathbf{x})+d(\mathbf{x}) x+\sum_{i} \beta_{i}(\mathbf{x}) y_{i} & -\beta_{1}(\mathbf{x}) y_{1} & -\beta_{2}(\mathbf{x}) y_{2} \\
-\beta_{1}(\mathbf{x}) y_{1} & \left(\beta_{1}(\mathbf{x})+\mu(\mathbf{x})\right) y_{1}+\nu \tilde{y} & -\nu \tilde{y} \\
-\beta_{2}(\mathbf{x}) y_{2} & -\nu \tilde{y} & \left(\beta_{2}(\mathbf{x})+\mu(\mathbf{x})\right) y_{2}+\nu \tilde{y}
\end{array}\right)
$$

where $\tilde{y}=y_{1}+y_{2}$. The vector $\mathbf{A}(\mathbf{x})$ and matrix $\mathbf{B}(\mathbf{x})$ of the Fokker-Planck equation (12) are associated with the system of stochastic differential equations (SDEs)

$$
d \mathbf{x}=\mathbf{A}(\mathbf{x}) d \tau+\Omega^{-1 / 2} \mathbf{C}(\mathbf{x}) d \mathbf{W}_{\tau},
$$

where $\mathbf{A}(\mathbf{x})=\left(\mathrm{A}_{1}(\mathbf{x}), \mathrm{A}_{2}(\mathbf{x}), \mathrm{A}_{3}(\mathbf{x})\right)^{T}, \mathbf{C}(\mathbf{x}) \mathbf{C}(\mathbf{x})^{T}=\mathbf{B}(\mathbf{x})$, and $d \mathbf{W}_{\tau}$ is a vector of $m$ independent Wiener processes, where $m$ is the number of columns of $\mathbf{C}(\mathbf{x})$ (this varies since the choice of $\mathbf{C}(\mathbf{x})$ is not unique, see Allen 2011). The relationship between the FokkerPlanck equation (12) and the system of SDEs given by (13) is a general relationship and is not specific to our model; this relationship is discussed in more detail elsewhere (Allen 2011; Gardiner 2009). Note that $\mathbf{A}(\mathbf{x})$ and $\mathbf{C}(\mathbf{x})$ are sometimes referred to as the 'drift' and 'diffusion' terms, respectively, of (13). If habitat size becomes large, $\Omega \rightarrow \infty$, stochasticity will disappear from (13) and we will be left with a system of ordinary differential equations (ODEs) given by $\dot{\mathbf{x}}=\mathbf{A}(\mathbf{x})$.

The pathogen strains will differ both in terms of their sterility virulence and (potentially) transmissibility. As such, we suppose that the fecundity of a host infected with strain $i$ is reduced by $\delta_{i}$ such that if $\delta_{i}=1$, strain $i$ completely sterilizes the host, whereas if $\delta_{i}=0$, strain $i$ causes no sterility virulence. We will assume that $\delta_{1}<\delta_{2}$, that is, strain 1 causes less sterility than strain 2 , but that strain 1 may have reduced transmissibility, $\beta_{1}(\mathbf{x})=\beta(\mathbf{x})(1-\varepsilon), \beta_{2}(\mathbf{x})=\beta(\mathbf{x})$, and that selection is weak, $0 \leq \varepsilon \ll 1$. Note that the assumption of weak selection places no restriction on between-strain differences in sterility virulence. We will also assume that mortality of both infected and uninfected hosts are non-decreasing functions of population density, that is, $\partial d / \partial \mathbf{x}_{i} \geq 0, \partial \mu / \partial \mathbf{x}_{i} \geq 0$.

Under these assumptions, (13) is equivalent to equations (1) from the main text. Let $\mathbf{A}(\mathbf{x})=\mathbf{A}(\mathbf{x} ; \varepsilon, \nu)$ (i.e., this just explicitly indicates that $\mathbf{A}(\mathbf{x})$ depends upon $\varepsilon$ and $\nu)$, then the ODE model $\dot{\mathbf{x}}=\mathbf{A}(\mathbf{x} ; \varepsilon, 0)$ predicts the strain with higher transmissibility (strain 2) will outcompete strain 1 and fix in the population, irrespective of the form of the transmission function, $\beta(\mathbf{x})$, or the between-strain difference in sterility virulence. If instead $\varepsilon=0$ and there is no fitness difference between the two strains, the strains will remain in their initial proportions, although the density of each strain may change. That is, if $y_{i}\left(t_{0}\right)$ and $y_{i}\left(t_{1}\right)$ are the densities of strain $i$ at time $t_{0}$ and $t_{1}$, then $\frac{y_{1}\left(t_{0}\right)}{y_{1}\left(t_{0}\right)+y_{2}\left(t_{0}\right)}=\frac{y_{1}\left(t_{1}\right)}{y_{1}\left(t_{1}\right)+y_{2}\left(t_{1}\right)}$. 


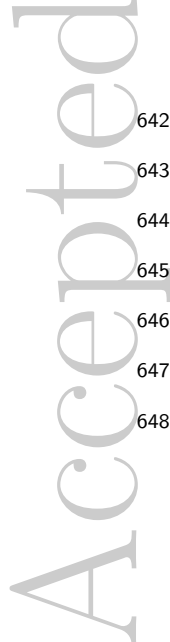

\section{Slow manifold approximation}

Assume that the ODE model $\dot{\mathbf{x}}=\mathbf{A}(\mathbf{x} ; 0,0)$ has a curve of endemic equilibria, $\gamma(w)=$ $\left(\gamma_{x}(w), \gamma_{1}(w), \gamma_{2}(w)\right)$, where $w$ is the variable we choose to parameterize the curve with (so one of the entries of $\gamma(w)$, i.e., $\gamma_{x}(w)$ or $\gamma_{i}(w)$ will be equal to $w$ ). If $\gamma(w)$ is globally asymptotically stable, trajectories of the ODE model $\dot{\mathbf{x}}=\mathbf{A}(\mathbf{x} ; 0,0)$ asymptotically approach different points on $\gamma(w)$. When $\Omega$ is large but finite, then when the system is far from $\gamma(w)$, the drift term will dominate (since $1 / \Omega$ is small), and so provided $\varepsilon$ and $\nu$ are also small, trajectories of system (13) will approach $\gamma(w)$ roughly along the flow lines of the ODE system $\dot{\mathbf{x}}=\mathbf{A}(\mathbf{x} ; 0,0)$. Once near $\gamma(w)$, however, although in system (13) $\mathbf{A}(\mathbf{x} ; \varepsilon, \nu)$ will become very small, $\mathbf{C}(\mathbf{x})$ will not. Thus stochasticity will dominate the system behaviour, causing the system to move along $\gamma(w)$ (now referred to as a slow manifold (Berglund and Gentz 2006; Parsons and Rogers 2017). Since we should reasonably expect the system (13) to approach $\gamma(w)$ before moving along it, we wish to derive an equation of motion for the stochastic system (13) along $\gamma(w)$ (see Figure $5 \mathbf{a}$ for an illustration).

As described in greater detail in Parsons and Rogers (2017), to do so we imagine (stochastic) trajectories along the slow manifold receiving random "kicks" displacing the system from the slow manifold to (say) point $\mathbf{x}_{w}=\left(w_{0}, w_{1}, w_{2}\right)$. Once the system is "kicked away" from the slow manifold, then provided $\Omega$ is large the deterministic portion of (13) dominates and so we may expect the system to rapidly return to the slow manifold along the (deterministic) flow lines. Provided $\nu$ and $\varepsilon$ are small, the new position on the manifold will be approximately determined by the solution of the system of differential equations $\dot{\mathbf{x}}=\mathbf{A}(\mathbf{x} ; 0,0)$ with initial conditions $\mathbf{x}_{w}$. Since for the ODE system $\dot{\mathbf{x}}=\mathbf{A}(\mathbf{x} ; 0,0)$ we have $\frac{d y_{2}}{d y_{1}}=\frac{y_{2}}{y_{1}}$, this will have solution $y_{2}=\left(w_{2} / w_{1}\right) y_{1}$ if $\mathbf{x}_{w}$ is the initial condition (position system was "kicked" too). Thus if a trajectory is initially at $\left(y_{1}, y_{2}\right)$ (say), then it will return to the point on the slow manifold given implicitly by

$$
\gamma_{2}(w)=\frac{y_{2}}{y_{1}} \gamma_{1}(w)
$$

Note that as we are going to treat the initial condition as a dynamic quantity, we have used the variables describing the density of strain 1 and 2 in equation (14) to denote the initial condition rather than the static initial condition $\mathbf{x}_{w}$.

From (14), since $w$ parameterizes the slow manifold, $d w / d t$ will reveal how the position of the system along the slow manifold evolves with time (Parsons and Rogers 2017). Let $\mathcal{G}(w)=\gamma_{2}(w) / \gamma_{1}(w)$ and (assuming $\mathcal{G}(w)$ is invertible) $g(w)=\mathcal{G}^{-1}(w)$. Hence from (14) we have $w=g\left(y_{2} / y_{1}\right)$, and applying Itô's formula (e.g., Allen (2011)) gives

$$
\begin{aligned}
& d w=\sum_{i=1}^{2}\left(\mathrm{~A}_{i+1}(\mathbf{x}) \frac{\partial g}{\partial y_{i}}+\frac{1}{2 \Omega} \sum_{j=1}^{2} \mathrm{~B}_{i+1 j+1}(\mathbf{x}) \frac{\partial^{2} g}{\partial y_{i} \partial y_{j}}\right) d \tau \\
&+\sqrt{\sum_{i=1}^{2} \sum_{j=1}^{2} \frac{\mathrm{B}_{i+1 j+1}(\mathbf{x})}{\Omega} \frac{\partial g}{\partial y_{i}} \frac{\partial g}{\partial y_{j}}} d W_{\tau}
\end{aligned}
$$

where the $\mathrm{A}_{i}(\mathbf{x})$ and $\mathrm{B}_{i j}(\mathbf{x})$ are from (12). Since $\varepsilon, \nu$, and $1 / \Omega$ are small, we should expect the dynamics of $w$ to remain "close" to the slow manifold, and so we approximate (15) by 
evaluating it along the slow manifold, that is $\mathbf{x}=\gamma(w)$. If we then let $\mathcal{I}(w)=\gamma_{1}(w)+\gamma_{2}(w)$ be the total density of infected individuals along the slow manifold, and $p=\gamma_{1}(w) / \mathcal{I}(w)$ be the fraction of infected hosts infected with strain 1, we can apply Itô's formula to compute

$$
d p=\underbrace{\left(\nu(1-2 p)-\varepsilon \beta\left(\gamma\left(w_{p}\right)\right) p(1-p)\right)}_{a(p)} d \tau+(\underbrace{\frac{p(1-p) \Re\left(w_{p}\right)}{\Omega}}_{\sigma^{2}(p)})^{1 / 2} d W_{\tau},
$$

where $w_{p}$ is the solution of $p=\gamma_{1}\left(w_{p}\right) / \mathcal{I}\left(w_{p}\right)$,

$$
\mathfrak{R}\left(w_{p}\right)=\frac{\beta\left(\gamma\left(w_{p}\right)\right)+\mu\left(\gamma\left(w_{p}\right)\right)}{\mathcal{I}\left(w_{p}\right)},
$$

and because $\varepsilon, \nu$ and $1 / \Omega$ are small, we have discarded terms of order $\varepsilon / \Omega$ and $\nu / \Omega$. If we let $\gamma_{x}\left(w_{p}\right)=x_{p}, \gamma\left(w_{p}\right)=\mathbf{x}_{p}$, and $t=\tau$ we obtain equation (3) of the main text.

Note that (16) is the same equation that we would have obtained if we had applied Itô's formula to $p=y_{1} /\left(y_{1}+y_{2}\right)$ (using (13)), before evaluating the result along the slow manifold. The intuitive reason for this is that if the system receives a stochastic "kick" away from the slow manifold to point $\mathbf{x}_{w}$ before returning along the flow lines of the ODE system $\dot{\mathbf{x}}=\mathbf{A}(\mathbf{x} ; 0,0)$ to the (new) position on the manifold, $\left(z_{0}, z_{1}, z_{2}\right)$, because the per capita growth rates of strain 1 and 2 are identical (when $\varepsilon=0$ ), we will necessarily have $w_{1} /\left(w_{1}+w_{2}\right)=z_{1} /\left(z_{1}+z_{2}\right)$, that is, the proportion of infected hosts infected with strain 1 remains constant along the flow lines of the ODE system.

To summarize, our starting point was the stochastic process given by (11). Then provided habitat size is large $(\Omega \gg 0)$, we showed that (11) can be approximated by a three-variable SDE system, (13). We then assumed that if mutations are rare and selection is weak $(\nu$ and $\varepsilon$ small), the three-variable SDE system (13) has a slow manifold of endemic equilibria, $\gamma(w)$. Because we expect that when $\nu, \varepsilon$, and $1 / \Omega$ are small, (13) will proceed rapidly to this slow manifold before moving along it, we then reduced the three-variable SDE system (13) to a single-variable SDE describing the stochastic dynamics along the slow manifold, equation (16), using the techniques of Parsons and Rogers 2017. Associated with the single-variable SDE (16) is a diffusion process with infinitesimal mean $a(p)$ and infinitesimal variance $\sigma^{2}(p)$ (Karlin and Taylor 1981). Using the standard tools of diffusion theory (e.g., Gardiner 2009; Karlin and Taylor 1981), we can compute the stationary distribution associated with (16), as well as time till absorption (i.e., time till fixation of one strain or another; this assumes $\nu=0)$ to verify the accuracy of (16) in approximating the full stochastic process given by (10). In Figure 5, we plot some examples.

\section{Evaluating which type is favoured by evolution}

We wish to understand which strain is stochastically favoured. We will take the approach that we used elsewhere (McLeod and Day 2019), and we will say that if the population is more likely to be observed in a state favourable to strain $i$, then strain $i$ is favoured. Our approach will be to focus upon the speed measure, $m(p)$, of the diffusion process with 

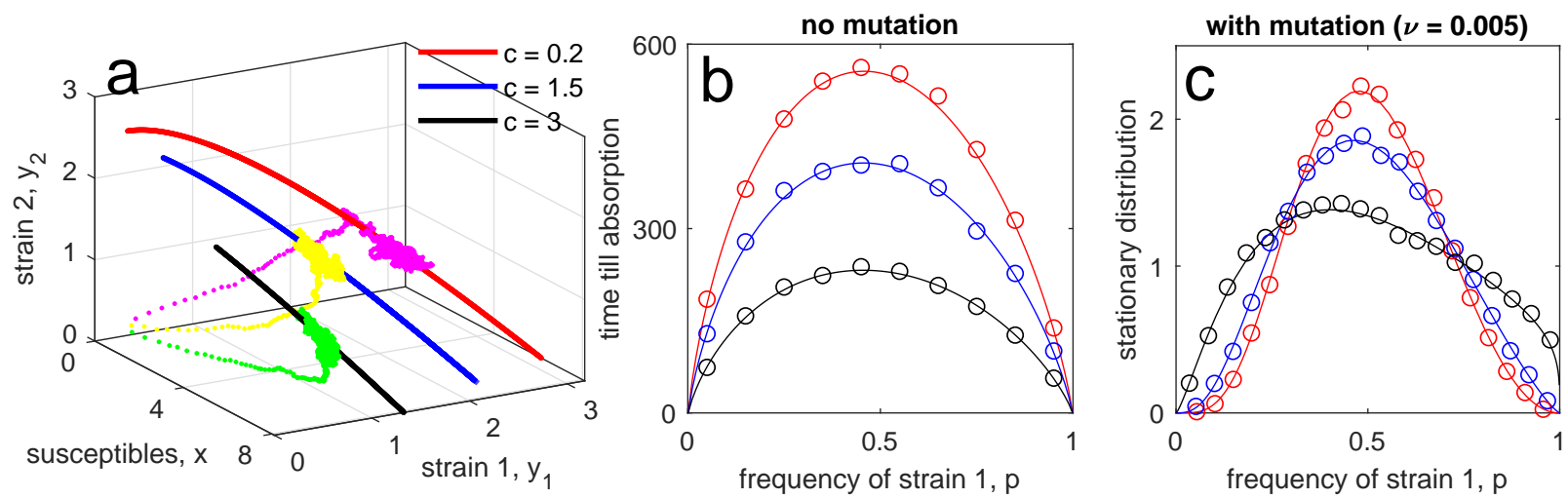

Figure 5: Behaviour of model $b(\mathbf{x})=b\left(x+y_{1}+0.25 y_{2}\right), d(\mathbf{x})=0.25+0.5\left(x+y_{1}+y_{2}\right)$, $\mu(\mathbf{x})=d(\mathbf{x})+0.25, \beta(\mathbf{x})=8 x /\left(x+y_{1}+y_{2}+c\right)$, with $\Omega=10^{3}$ and $\varepsilon=0$. In all subplots, we vary the value of $c$ from $c=0.2$ (red) to $c=1.5$ (blue) to $c=3$ (black). In subplot a, the solid curves are the slow manifold, while the scattered trajectories are simulations of the full stochastic process defined by (10) using Gillespie's algorithm (Gillespie 1977). The system rapidly moves to the vicinity of the slow manifold, before drifting along it; it is this motion described by (16). In subplot $\mathbf{b}$, we suppose there is no mutation, $\nu=0$, and calculate the time until one strain goes to fixation. The curves are the analytic prediction from (16), whereas each circle is the average of 2000 simulations of (10) using Gillespie's algorithm, initialized at the point on the slow manifold given by the particular value of $p$. Finally, in subplot c, we suppose there is mutation, $\nu=0.005$, and compute the stationary distribution. The curves are the analytic prediction from (16), whereas the circles are $2 \times 10^{4}$ simulations of (10) using Gillespie's algorithm, where each simulation was run for $3 \times 10^{4}$ time units from the arbitrarily chosen initial condition $\left(X, Y_{1}, Y_{2}\right)=(200,200,200)$.

infinitesimal mean $a(p)$ and variance $\sigma^{2}(p)$, defined as

$$
m(p) \equiv \frac{1}{\sigma^{2}(p)} \exp \left(2 \int^{p} \frac{a(z)}{\sigma^{2}(z)} d z\right) .
$$

If the integral $\int_{0}^{1} m(p) d p$ exists, then the diffusion process admits a stationary distribution, $C_{0} m(p)$, where $C_{0}=1 / \int_{0}^{1} m(p) d p$ (Etheridge 2012; Karlin and Taylor 1981). This occurs if mutation rate is sufficiently high. However, even if no stationary distribution exists, $m\left(p_{0}\right)(\Delta p)^{2}$ is proportional to the time it takes a process initially at $p_{0}$ to exit the interval $\left(-\Delta p+p_{0}, p_{0}+\Delta p\right)$ (Ewens 2004; Karlin and Taylor 1981). Hence as $m\left(p_{0}\right)$ increases for a given $p_{0}$, all else being equal, the process is more likely to be observed in state $p_{0}$.

To make progress in understanding $m(p)$, we will use the fact that $\varepsilon$ is small to approximate $m(p)$. To do so, suppose that $\delta_{1}=\delta-\omega \varepsilon$ and $\delta_{2}=\delta$ (recall that $\delta_{i}$ corresponds to the sterility virulence of strain $i$ such that if $\delta_{i}=0$ strain $i$ causes no sterility virulence). This implies that $0 \leq \omega \varepsilon \leq \delta$. Then since $\beta_{1}(\mathbf{x})=\beta(\mathbf{x})(1-\varepsilon)$ and $\beta_{2}(\mathbf{x})=\beta(\mathbf{x})$, this implies strain 1 decreases its transmission by a factor $\varepsilon$, but by doing so it decreases its sterility virulence by a factor $\omega \varepsilon$, where $\omega$ is a scaling factor that relates between-strain differences in transmission to between-strain differences in sterility virulence. With this in mind, we 
will write $m(p ; \varepsilon)$ to explicitly show the dependence of the speed measure on $\varepsilon$. A Taylor expansion of $m(p ; \varepsilon)$ in $\varepsilon$ gives

$$
m(p ; \varepsilon) \approx m(p ; 0)+\left.\frac{\partial m}{\partial \varepsilon}\right|_{\varepsilon=0} \varepsilon+\mathcal{O}\left(\varepsilon^{2}\right)
$$

so we need to compute $m(p ; 0)$ and $\left.\frac{\partial m}{\partial \varepsilon}\right|_{\varepsilon=0}$.

If $\varepsilon=0$, then $\beta_{1}(\mathbf{x})=\beta_{2}(\mathbf{x})$ and $\delta_{1}=\delta_{2}$, and so the two strains are mathematically identical. It follows that the density of susceptibles along the slow manifold is constant and so $\mathfrak{R}\left(w_{p}\right)=\mathfrak{R}$, that is, $\mathfrak{R}$ is constant with respect to $p$. Hence

$$
m(p ; 0)=\frac{\Omega}{p(1-p) \Re} \exp \left(2 \int^{p} \frac{\nu(1-2 z) \Omega}{z(1-z) \mathfrak{R}} d z\right)=\frac{\Omega}{\Re}(p[1-p])^{\frac{2 \nu \Omega}{\Re}-1} .
$$

Similarly,

$$
\left.\frac{\partial m}{\partial \varepsilon}\right|_{\varepsilon=0}=m(p ; 0)\left[-\frac{1}{\mathfrak{R}} \frac{d \mathfrak{R}}{d w_{p}} \frac{d w_{p}}{d \varepsilon}-\frac{2 \Omega}{\mathfrak{R}} \int^{p}\left(\beta\left(w_{p}\right)+\frac{\nu(1-2 z)}{z(1-z) \mathfrak{R}} \frac{d \mathfrak{R}}{d w_{p}} \frac{d w_{p}}{d \varepsilon}\right) d z\right]_{\varepsilon=0} .
$$

To compute the integral in (20), we need to know more about $\frac{d \Re}{d w_{p}} \frac{d w_{p}}{d \varepsilon}$. On the slow manifold, there are no selective differences between strains, and so $\beta_{1}\left(w_{p}\right)=\beta_{2}\left(w_{p}\right)$. However, the variable $w_{p}$, which is a function of $p$, will not be constant with respect to $p$ because of the role played by the difference in sterility virulence between strains. Since the difference in sterility virulence between strains is the only thing distinguishing strain 1 from strain 2, we can write $w_{p}$ as a function of the expected level of sterility in the population, $\delta_{1} p+\delta_{2}(1-p)$, and so using our earlier notation, $w_{p}\left(\delta_{1} p+\delta_{2}(1-p)\right)=w_{p}([\delta-\omega \varepsilon] p+\delta[1-p])=w_{p}(\delta-\omega \varepsilon p)$. It follows that

$$
\left.\frac{d \mathfrak{R}}{d w_{p}} \frac{d w_{p}}{d \varepsilon}\right|_{\varepsilon=0}=-\left.\frac{d \mathfrak{R}}{d w_{p}} \frac{d w_{p}}{d \delta}\right|_{\varepsilon=0} \omega p=C \omega p
$$

where $C \equiv-\left.\frac{d \Re}{d w_{p}} \frac{d w_{p}}{d \delta}\right|_{\varepsilon=0}=-\frac{d \Re}{d \delta}$ is a constant that does not depend upon $p$ (or $\varepsilon$ ). Hence $C$ is the change in $\mathfrak{R}$ due to between-strain differences in sterility virulence: if $C<0$, then strain 1 minimizes $\mathfrak{R}\left(w_{p}\right)$, whereas if $C>0$, then strain 2 minimizes $\mathfrak{R}\left(w_{p}\right)$. Using this information in (20) gives

$$
\left.\frac{\partial m}{\partial \varepsilon}\right|_{\varepsilon=0}=-m(p ; 0) \frac{C \omega}{\mathfrak{R}}\left(\frac{2 \Omega}{C \omega}\left[\beta(w)+\frac{C \omega}{2 \Omega}\right] p+\frac{2 \nu \Omega}{\mathfrak{R}}(2 p+\ln (1-p))\right) .
$$

Hence

$$
m(p ; \varepsilon) \approx \frac{\Omega}{\mathfrak{R}}(p[1-p])^{a_{1}-1}\left[1-\varepsilon \frac{C \omega}{\mathfrak{R}}\left(a_{0} p+a_{1}(2 p+\ln (1-p))\right)\right]+\mathcal{O}\left(\varepsilon^{2}\right)
$$

where $a_{0}=\frac{2 \Omega}{C \omega}\left[\beta(w)+\frac{C \omega}{2 \Omega}\right]$ and $a_{1}=\frac{2 \nu \Omega}{\Re}(w$ is the density of susceptibles when $\varepsilon=0$ and so does not depend upon $p$ ).

Now how can we use this approximation of $m(p ; \varepsilon)$ to understand the evolution of sterility virulence? Observe that mutation rate, $\nu$, appears only in $a_{1}$ and nowhere else in $m(p ; \varepsilon)$. 
Hence as we vary $\nu$, we can vary $a_{1}$ independent of all other factors in (22). Doing so will have a substantial qualitative effect upon the behaviour of (22). As a result, we need to divide our analysis of (22) into three mutation regimes: (i) low or negligible mutation rate, (ii) intermediate mutation rate, and (iii) high mutation rate. Although we will define these mutational regimes more precisely in what follows, we observe that our 'high' mutation rate is a mutation rate sufficiently large such that a normalizable stationary distribution exists, but also sufficiently small such that the approximation given by (16) is still valid. In what follows, we consider each in turn.

\subsection{Low mutation rate}

Suppose mutation rate is sufficiently low such that we are dealing with a sequential-fixation process. In such a process, whenever a mutant strain appears in the population, that strain either goes extinct or fixes in the population before another mutation can occur. A standard approach to dealing with such a process is to construct a Markov chain on the state space of possible strain types (e.g., McCandlish and Stoltzfus (2014)). In particular, let $\nu_{i j}$ denote the per capita rate at which strain $i$ mutates to strain $j$, and $N_{i}$ be the (expected) number of hosts infected with strain $i$ at equilibrium in a population monomorphic for strain $i$. Then if $\phi_{i j}$ is the probability a single strain $j$ mutant fixes in a population otherwise monomorphic for strain $i$, the population will transition from a population monomorphic for strain $i$ to a population monomorphic for strain $j$ at rate

$$
\left(\begin{array}{c}
\text { rate of } \\
\text { mutations }
\end{array}\right) \times\left(\begin{array}{c}
\text { fixation } \\
\text { probability }
\end{array}\right)=\nu_{i j} N_{i} \times \phi_{i j}
$$

If selection is sufficiently weak (and mutations are negligible), then $a(p) \approx 0$. Hence the diffusion process defined by $a(p)$ and $\sigma^{2}(p)$ is on its natural scale, and so the fixation probability of any strain is equal to its proportion in the population. But then $\nu_{i j} N_{i} \times \phi_{i j}=\nu_{i j} N_{i} \times\left(1 / N_{i}\right)=\nu_{i j}$. And so in the absence of any mutational biases, the Markov chain is equally likely to be in any particular quasi-fixation state, a standard result for neutral and nearly-neutral processes (Kimura 1968; King and Jukes 1969; McCandlish and Stoltzfus 2014).

What we have failed to take into account is that the level of sterility virulence will alter the time spent transitioning between quasi-fixation states by modifying the population dynamics. This can be understood by considering (22). Since mutations are small, $\nu \rightarrow 0$, this implies that $a_{1} \rightarrow 0$ as well. And so in (22) we are left with

$$
m(p ; \varepsilon) \approx m(p ; 0)\left[1-\varepsilon \frac{C \omega}{\mathfrak{R}} a_{0} p\right]+\mathcal{O}\left(\varepsilon^{2}\right)
$$

As $m(p ; 0)=m(1-p ; 0)$ (i.e., $m(p ; 0)$ is symmetric about $p=1 / 2)$, and $\varepsilon \omega / \mathfrak{R}>0$, if $C a_{0}<0$, then $m(1-p ; \varepsilon)>m(p ; \varepsilon)$ for all $p \in(0,1 / 2)$, and so the process will spend more time in states favourable to strain 1 . Using our definition of $a_{0}$ provided immediately after equation (22), if

$$
-\frac{C \omega}{2 \Omega}>\beta(w)
$$

then $C a_{0}<0$. Hence if $(24)$ is satisfied, then strain 1 is stochastically favoured. Note that in 
the absence of a (positive) link between transmission and sterility virulence, that is, $\varepsilon=0$, then since $m(p ; \varepsilon) \approx 1 /\left[\sigma^{2}(p)\right]$, we see that provided $\mathfrak{R}\left(w_{p}\right)$ is monotonic in $p$, whichever strain minimizes $\mathfrak{R}\left(w_{p}\right)$ is favoured. When the two strains are "similar" this is equivalent to considering the sign of $C$.

\subsection{Intermediate mutation rate}

We define an intermediate mutation rate to be a mutation rate which is not negligible, but is also not sufficiently high such that a stationary distribution exists (i.e., $\int_{0}^{1} m(p ; \varepsilon) d p$ does not exist). From consideration of $m(p ; 0)$, we see that if $a_{1}<1$, that is, $\mu<\mathfrak{R} /[2 \Omega]$, then a stationary distribution will not exist. When mutation rate is intermediate, determining which strain is favoured becomes complex. This can be most easily seen by considering whether the quantity $m(1-p ; \varepsilon)-m(p ; \varepsilon)$ is positive or negative. If for a given $p_{0}$ it is positive, this means the process takes longer to exit a region around $1-p_{0}$ then $p_{0}$, and so we may argue that for that particular value of $p_{0}$ strain 1 is favoured. Using our previously calculated relations, and making use of the fact $m(p ; 0)=m(1-p ; 0)$, yields

$$
m(1-p ; \varepsilon)-m(p ; \varepsilon)=-m(p ; 0) \frac{\varepsilon C \omega}{\mathfrak{R}}\left(\left(a_{0}+2 a_{1}\right)(1-2 p)+a_{1} \ln \left(\frac{p}{1-p}\right)\right) .
$$

We know that $a_{1} \in(0,1)$ for intermediate mutation rates, while by inspection, $\lim _{p \rightarrow 0}[m(1-$ $p ; \varepsilon)-m(p ; \varepsilon)]=\operatorname{sign}(C) \infty$. Moreover, if $a_{0}>0$, then for some $p^{*} \in(0,1 / 2), m\left(1-p^{*} ; \varepsilon\right)-$ $m\left(p^{*} ; \varepsilon\right)=0$. If such a $p^{*}$ exists, then as $a_{1} \rightarrow 0, p^{*} \rightarrow 0$. Using this information, we see that if $C>0$ (and so $a_{0}>0$ ), then for $p<p^{*}(25)$ is positive, whereas for $p>p^{*}$, (25) is negative. If instead $C<0$, then if (24) is satisfied, $a_{0}>0$, otherwise $a_{0} \leq 0$. If $a_{0} \leq 0$, then (25) is negative for all $p \in(0,1 / 2)$. Conversely, if $a_{0}>0$, then for $p<p^{*}$, (25) is negative, whereas for $p>p^{*}$, it is positive.

Although the preceding analysis is somewhat abstract, we can understand it biologically by observing that there exist essentially two regimes based upon the population composition: either the population is mainly monomorphic, or it is polymorphic. If the population is mainly monomorphic, then whichever strain maximizes $\mathfrak{R}\left(w_{p}\right)$ is favoured. This is because for mainly monomorphic populations, the two competing forces are mutations, which push the population to a polymorphic state, and genetic drift, which is proportional to $\mathfrak{R}\left(w_{p}\right)$, and keeps the population in a monomorphic state. If $C<0$, then strain 1 maximizes $\mathfrak{R}\left(w_{p}\right)$, and so is favoured in the monomorphic regime, whereas if $C>0$, strain 2 maximizes $\mathfrak{R}\left(w_{p}\right)$ and so is favoured in the monomorphic regime. If instead the population is polymorphic, then when inequality (24) is satisfied, strain 1 is favoured; whereas if it is not satisfied, strain 2 is favoured. The breakpoint at which the population switches from the mainly monomorphic regime to the polymorphic regime occurs at $p=p^{*}$. Hence decreasing mutation rate will increase the range of $p$ that constitutes the polymorphic regime. If there is no (positive) link between transmissibility and sterility virulence, $\varepsilon=0$, then provided $\mathfrak{R}\left(w_{p}\right)$ is monotonic in $p$, whichever strain minimizes $\mathfrak{R}\left(w_{p}\right)$ is favoured when $p>p^{*}$, whereas whichever strain maximizes $\mathfrak{R}\left(w_{p}\right)$ is favoured for $p<p^{*}$.

Thus for an intermediate mutation rate, if there is sufficient "segregating variation" so that the population is in the polymorphic state, then if there is a positive link between transmission and sterility virulence, if (24) is satisfied then strain 1 is favoured, whereas if there is no transmission-sterility virulence trade-off, then whichever type minimizes $\mathfrak{R}\left(w_{p}\right)$ 
is favoured.

\subsection{High mutation rate}

If mutation rate is sufficiently high such that $a_{1}>1$, then from $(22)$ the integral $\int_{0}^{1} m(p ; \varepsilon) d p$ exists. Consequently, a stationary distribution, $\pi(p ; \varepsilon) \equiv C_{0}(\varepsilon) m(p ; \varepsilon)$ also exists, where $C_{0}(\varepsilon)=1 / \int_{0}^{1} m(p ; \varepsilon) d p$. Because our objective is to determine whether a particular strain is more or less likely to be observed in the population, when a stationary distribution exists we will say that if

$$
\int_{1 / 2}^{1} \pi(p ; \varepsilon) d p>\frac{1}{2}
$$

then strain 1 (the less sterilizing strain) is favoured. In words, inequality (26) means that the population is more likely to be observed in a state where strain 1 is present in greater frequency.

Since $\varepsilon$ is small, and because $\int_{1 / 2}^{1} \pi(p ; 0) d p=1 / 2$, a Taylor expansion gives

$$
\int_{1 / 2}^{1} \pi(p ; \varepsilon) d p \approx \frac{1}{2}+\frac{\varepsilon C_{0}(0)}{2}\left[\int_{1 / 2}^{1} \frac{\partial m}{\partial \varepsilon} d p-\int_{0}^{1 / 2} \frac{\partial m}{\partial \varepsilon} d p\right]_{\varepsilon=0}+\mathcal{O}\left(\varepsilon^{2}\right) .
$$

Hence strain 1 will be favoured if

$$
m_{\mathrm{int}} \equiv\left[\int_{1 / 2}^{1} \frac{\partial m}{\partial \varepsilon} d p-\int_{0}^{1 / 2} \frac{\partial m}{\partial \varepsilon} d p\right]_{\varepsilon=0}>0 .
$$

Inserting (21), we obtain $m_{\text {int }}=-\frac{\Omega C \omega}{\mathfrak{R}^{2}} \Theta\left(a_{0}, a_{1}\right)$ where

$$
\begin{aligned}
\Theta\left(a_{0}, a_{1}\right) & \equiv a_{0}\left[\int_{1 / 2}^{1} p^{a_{1}}(1-p)^{a_{1}-1} d p-\int_{0}^{1 / 2} p^{a_{1}}(1-p)^{a_{1}-1} d p\right] \\
& +a_{1}\left[\int_{1 / 2}^{1}(p[1-p])^{a_{1}}(2 p+\ln (1-p)) d p-\int_{0}^{1 / 2}(p[1-p])^{a_{1}}(2 p+\ln (1-p)) d p\right] .
\end{aligned}
$$

Because $\Theta\left(a_{0}, a_{1}\right)$ is a function of two variables, it can be plotted in terms of $a_{0}$ and $a_{1}$ (Fig. $6)$. Since $a_{1}=\frac{2 \Omega \nu}{\mathfrak{R}}$, while $a_{0}=1+\frac{\beta(w)}{C \omega /[2 \Omega]}$; varying $a_{1}$ and $a_{0}$ independently as in Figure 6 means we are manipulating mutation rate (for $a_{1}$ ) and the ratio $\frac{\beta(w)}{C \omega /[2 \Omega]}$ (for $a_{0}$ ).

To understand Figure 6, observe that if there is no link between transmission and sterility virulence $(\varepsilon=0)$, then $a_{0}=1$. Hence if $C>0$, then $m_{\text {int }}<0$, whereas if $C<0$ then $m_{\text {int }}>0$, and so whichever strain minimizes $\mathfrak{R}\left(w_{p}\right)$ is favoured (provided $\mathfrak{R}\left(w_{p}\right)$ is monotonic in $p$ ). If there is a (positive) link between transmission and sterility, then if $C>0$ (so strain 2 minimizes $\left.\mathfrak{R}\left(w_{p}\right)\right)$, we have $a_{0} \in(1, \infty)$ and so $m_{\text {int }}<0$ and strain 2 is favoured. If instead $C<0$ and there is a (positive) link between transmission and sterility, then there are two cases. If (24) is not satisfied, then $a_{0}<0$ and so strain 2 is favoured. If (24) is satisfied, then $a_{0} \in(0,1)$. So we see that the more $-\frac{C \omega}{2 \Omega}$ exceeds $\beta(w)$, the closer $a_{0}$ gets to 1 and so the likelihood that strain 1 is favoured increases. However, the precise breakpoint at which 


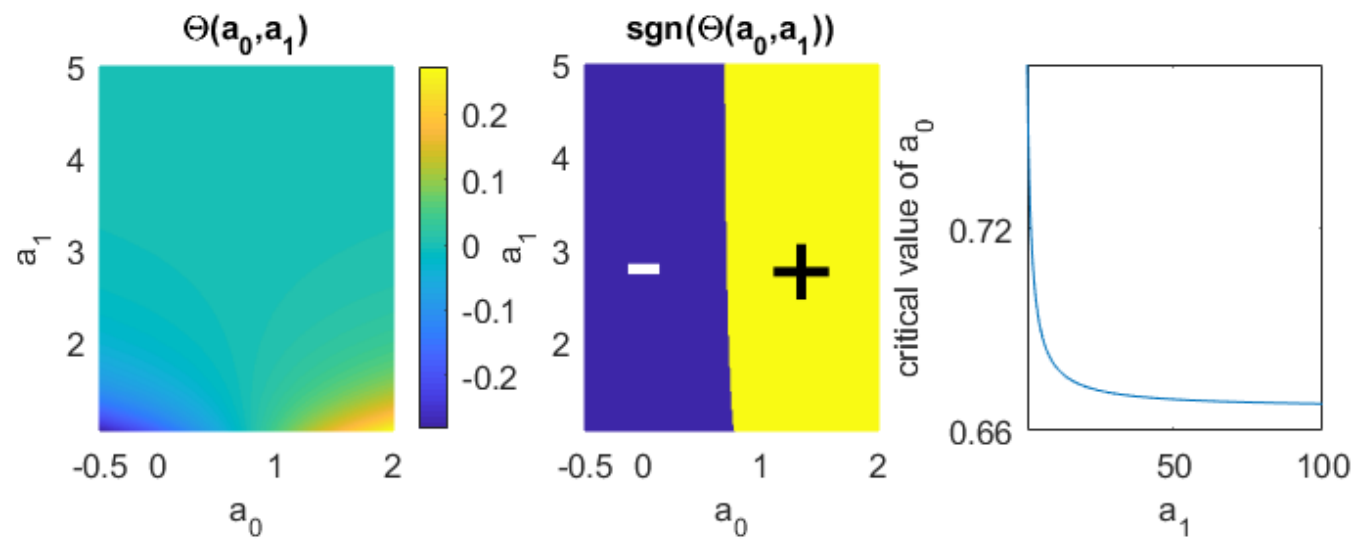

Figure 6: Change in $\Theta\left(a_{0}, a_{1}\right)$ as we manipulate $a_{0}$ and $a_{1}$. Since $a_{0}=1+\frac{\beta(w)}{C \omega /[2 \Omega]}$ and $a_{1}=\frac{2 \Omega \nu}{\Re}, a_{0}$ can be considered a proxy for $\frac{\beta(w)}{C \omega /[2 \Omega]}$ while $a_{1}$ is a proxy for mutation rate $\nu$.

this occurs at will depend upon $a_{1}$, and so mutation rate, as Figure 6 reveals.

To summarize our results for the three different mutation regimes, we have shown the following. If there is no link between sterility virulence and transmission, $\varepsilon=0$, then provided $\mathfrak{R}\left(w_{p}\right)$ is monotonic in $p$, whichever type minimizes $\mathfrak{R}\left(w_{p}\right)$ is favoured (with the caveat that for intermediate mutation rates we are only considering polymorphic populations). If there instead is a weak transmission-sterility trade-off, then in order for strain 1 to be favoured it is necessary that inequality (24) be satisfied (which at minimum requires strain 1 to minimize $\left.\mathfrak{R}\left(w_{p}\right)\right)$. If inequality $(24)$ is satisfied and mutation rate is low, then this is sufficient to favour strain 1, but as mutation rate increases the results become more complex. However, as a general rule of thumb, the more $-\frac{C \omega}{2 \Omega}$ exceeds $\beta(w)$, the greater the likelihood that strain 1 will be favoured.

\section{How does epidemiology affect $\mathfrak{R}\left(w_{p}\right)$ ?}

The results of the previous section demonstrate the importance of understanding how epidemiology affects $\mathfrak{R}\left(w_{p}\right)$. In particular, in the absence of selection $(\varepsilon=0)$, whichever strain minimizes $\mathfrak{R}\left(w_{p}\right)$ will be favoured by demographic stochasticity. When there is selection, then for stochasticity to overcome selection and favour strain 1 , we require at minimum that

$$
-\frac{C \omega}{2 \Omega}>\beta(w)
$$

which necessarily implies that strain 1 must minimize $\mathfrak{R}\left(w_{p}\right)$.

Hence our focus here will be to determine what level of sterility virulence (value of $\delta$ ) minimizes $\mathfrak{R}\left(w_{p}\right)$. To answer this, it suffices to consider the one strain ODE system

$$
\begin{aligned}
& \dot{x}=b(x, y, \delta)-d(x, y) x-\beta(x, y) y, \\
& \dot{y}=(\beta(x, y)-\mu(x, y)) y .
\end{aligned}
$$

Note that we have explicitly indicated the dependence of birth rate, $b(x, y, \delta)$ upon the 
sterility parameter $\delta$. Suppose we are at the locally asymptotically stable (LAS) endemic equilibrium, $(\bar{x}, \bar{y})$, which is the solution to $\dot{x}=\dot{y}=0$ satisfying $\bar{x}, \bar{y}>0$ (note that in the main text, we used $\overline{\mathcal{I}}$ instead of $\bar{y})$. How does a change in $\delta$ affect the endemic equilibrium? Let $r(x, y)=\beta(x, y)-\mu(x, y)$ denote per capita growth of the pathogen. Then implicit differentiation and some rearrangement yields

$$
\frac{d \bar{x}}{d \delta}=-\left.\Gamma(x, y) \frac{\partial r(x, y)}{\partial y}\right|_{\substack{x=\bar{x} \\ y=\bar{y}}} \text { and } \frac{d \bar{y}}{d \delta}=\left.\Gamma(x, y) \frac{\partial r(x, y)}{\partial x}\right|_{\substack{x=\bar{x} \\ y=\bar{y}}}
$$

where

$$
\Gamma(x, y)=\frac{\partial b}{\partial \delta} /\left(\frac{\partial \dot{x}}{\partial x} \frac{\partial r}{\partial y}-\frac{\partial \dot{x}}{\partial y} \frac{\partial r}{\partial x}\right) .
$$

When $\Gamma(x, y)$ is evaluated at the endemic equilibrium, $(\bar{x}, \bar{y})$, the denominator is proportional to the determinant of the Jacobian of (28) evaluated at the endemic equilibrium. As the endemic equilibrium is assumed to be LAS, the denominator is therefore positive and since we have assumed that whenever the pathogen is present, $\frac{\partial b}{\partial \delta}<0$, we can conclude $\Gamma(x, y)$ is negative. Since mortality is a non-decreasing function of density, $\partial \mu / \partial y \geq 0$, and as we should expect per capita transmission to be a non-increasing function of the density of infected individuals, $\partial \beta / \partial y \leq 0$, it follows that $\partial r(x, y) / \partial y \leq 0$ and so $d \bar{x} / d \delta \leq 0$. However, we cannot conclude anything about the sign $d \bar{y} / d \delta$ since we do not know the sign of $\partial r / \partial x$.

Now, consider $\mathfrak{R}\left(w_{p}\right)$. Because we are dealing with the one-strain case, we have $\mathfrak{R}(\bar{x}, \bar{y})=$ $\frac{2 \beta(\bar{x}, \bar{y})}{\bar{y}}$, where we have used that $\beta(\bar{x}, \bar{y})=\mu(\bar{x}, \bar{y})$. We wish to understand how $\mathfrak{R}(\bar{x}, \bar{y})$ changes as we vary $\delta$. Differentiating with respect to $\delta$ gives

$$
\begin{aligned}
\frac{d}{d \delta} \mathfrak{R}(\bar{x}, \bar{y}) & \left.\left.=\frac{2}{\bar{y}}\left[\frac{\partial \beta}{\partial x} \frac{d \bar{x}}{d \delta}+\frac{\partial \beta}{\partial y} \frac{d \bar{y}}{d \delta}-\frac{\beta(x, y)}{y} \frac{d \bar{y}}{d \delta}\right]_{\substack{x=\bar{x} \\
y=\bar{y}\\
}} \frac{2 \Gamma}{\partial x}\right]_{\substack{x=\bar{x} \\
y=\bar{y}}}\left[-\frac{\partial \beta}{\partial x} \frac{\partial r}{\partial y}+\left(\frac{\partial \beta}{\partial y}-\frac{\beta(x, y)}{y}\right) \frac{\partial r}{y}\right)\left(\frac{\partial \beta}{\partial x}-\frac{\partial \mu}{\partial x}\right)\right]_{\substack{x=\bar{x} \\
y=\bar{y}}} \\
& =\frac{2 \Gamma}{\bar{y}}\left[-\frac{\partial \beta}{\partial x}\left(\frac{\partial \beta}{\partial y}-\frac{\partial \mu}{\partial y}\right)+\left(\frac{\partial \beta}{\partial y}-\frac{\beta(x, y)}{y}\right)\right. \\
& =\frac{2 \Gamma}{\bar{y}}\left[\frac{\partial \beta}{\partial x}\left(\frac{\partial \mu}{\partial y}-\frac{\beta(x, y)}{y}\right)-\frac{\partial \mu}{\partial x}\left(\frac{\partial \beta}{\partial y}-\frac{\beta(x, y)}{y}\right)\right]_{\substack{x=\bar{x} \\
y=\bar{y}}}
\end{aligned}
$$

Letting $\beta(x, y)=B x /(x+y+c)$ gives, after some simplifications

$$
\frac{d \Re}{d \delta}=\frac{2 \Gamma}{\bar{y}} \frac{\beta(\bar{x}, \bar{y})}{\bar{y} \bar{x}}\left[\frac{x+y}{x+y+c} x \frac{\partial \mu}{\partial x}+\frac{y+c}{x+y+c}\left(x \frac{\partial \mu}{\partial x}+y \frac{\partial \mu}{\partial y}-\mu(x, y)\right)\right]_{\substack{x=\bar{x} \\ y=\bar{y}}} .
$$

The term in the square brackets in (30) multiplied by -1 is what is presented in the main text; and as observed there, this shares the same sign as $d \mathfrak{R} / d \delta$ since $2 \Gamma \beta(\bar{x}, \bar{y}) /\left[\bar{x} \bar{y}^{2}\right]<0$ (because $\Gamma<0)$. 


\subsection{The variance in per capita growth is a non-increasing function of sterility virulence}

The other thing to show is that the variance in per capita growth of the pathogen, $\beta(\mathbf{x})+\mu(\mathbf{x})$, is a non-increasing function of sterility virulence. To do so, first suppose that mortality is density-independent. Then $\mu(\mathbf{x})$ is a constant, and so the variance in per capita growth is unaffected by sterility virulence, and so is trivially a non-increasing function of sterility virulence. Suppose instead that pathogen mortality is affected by sterility virulence. If we then implicitly differentiate $\beta(\bar{x}, \bar{y}(\bar{x}))-\mu(\bar{x}, \bar{y}(\bar{x}))=0$ and solve for $d \bar{y} / d \bar{x}$ we obtain

$$
\frac{d \bar{y}}{d \bar{x}}=\frac{\frac{\partial \beta}{\partial \bar{x}}-\frac{\partial \mu}{\partial \bar{x}}}{\frac{\partial \mu}{\partial \bar{y}}-\frac{\partial \beta}{\partial \bar{y}}}
$$

Then since $\beta(\bar{x}, \bar{y})+\mu(\bar{x}, \bar{y})=2 \mu(\bar{x}, \bar{y})$, we have

$$
\frac{d[2 \mu(\bar{x}, \bar{y})]}{d \delta}=2\left(\frac{\partial \mu}{\partial \bar{x}}+\frac{\partial \mu}{\partial \bar{y}} \frac{d \bar{y}}{d \bar{x}}\right) \frac{d \bar{x}}{d \delta}=2\left(\frac{\frac{\partial \mu}{\partial \bar{y}} \frac{\partial \beta}{\partial \bar{x}}-\frac{\partial \mu}{\partial \bar{x}} \frac{\partial \beta}{\partial \bar{y}}}{\frac{\partial \mu}{\partial \bar{y}}-\frac{\partial \beta}{\partial \bar{y}}}\right) \frac{d \bar{x}}{d \delta} .
$$

As $d \bar{x} / d \delta<0, \frac{\partial \beta}{\partial x}>0, \frac{\partial \beta}{\partial y} \leq 0$, and $\frac{\partial \mu}{\partial x}, \frac{\partial \mu}{\partial y}>0$, it follows that the variance in per-capita growth is a non-increasing function of sterility virulence.

\subsection{What happens when the population is strongly regulated by density-dependent fecundity and mortality is only weakly density- dependent?}

The analysis of (30) suggests that in order for sterility virulence to be stochastically favoured by frequency-dependent transmission, the 'strength' of density-dependent mortality cannot be too weak. However, many populations experiencing density-dependent mortality also experience density-dependent fecundity. Thus it is of interest to know what happens when density-dependent mortality is weak and the population is primarily regulated by densitydependent reproduction.

To gain some insight into this question, suppose $\mu(\mathbf{x})=m+\alpha+\kappa\left(x+\sum_{i} y_{i}\right)$, where $m$ and $\alpha$ are natural- and virulence-related mortality, and $\kappa$ controls the magnitude of densitydependent mortality. Then we can compute

$$
\frac{d \Re}{d \delta}=2\left(\frac{\kappa}{\overline{\mathcal{I}}(\bar{x})}-\frac{\kappa \bar{x}+m}{\overline{\mathcal{I}}(\bar{x})^{2}} \frac{d \overline{\mathcal{I}}}{d \bar{x}}\right) \frac{d \bar{x}}{d \delta}
$$

where we have left the equilibrium density of infected individuals as a function of the equilibrium density of susceptibles. Since $d \bar{x} / d \delta<0$, if density-dependent mortality is very weak, $\kappa \approx 0$, then $d \Re / d \delta$ has the same sign as $d \overline{\mathcal{I}} / d \bar{x}$. Solving $\beta(\mathbf{x})=\mu(\mathbf{x})$ for the equilibrium density of infected individuals, $\overline{\mathcal{I}}(\bar{x})$, computing $d \overline{\mathcal{I}} / d \bar{x}$, and then performing a Taylor expansion in $\kappa$ gives

$$
\frac{d \mathcal{I}}{d \bar{x}}=-1+\frac{B}{m}-\left(\frac{2 B \bar{x}}{m^{3}}-\frac{c}{m^{2}}\right) \kappa+\mathcal{O}\left(\kappa^{2}\right) .
$$


In agreement with our previous analysis, we can see from (33) and (34) that as transmission becomes increasingly frequency-dependent $(c \rightarrow 0)$, the likelihood that sterility virulence is favoured increases (i.e., it is more likely that $d \mathcal{I} / d \bar{x}<0$ ).

Indeed, if $c=0$ and there is no density-dependent fecundity, then the total population size must be regulated by density-dependent mortality, since pathogens exhibiting frequencydependent transmission cannot regulate population size (Getz and Pickering 1983). In this circumstance, as $\kappa$ becomes small, we would expect that $\bar{x}$ will become very large, and so we would generally expect $d \mathcal{I} / d \bar{x}<0$ due to the negative contribution of the coefficient multiplying $\kappa$.

However, if the population is strongly regulated by density-dependent fecundity, then as we decrease $\kappa$ we will have little effect upon $\bar{x}$. Thus the influence of the first-order term in $\kappa$ in (34) upon the sign of $d \mathcal{I} / d \bar{x}$ is diminished, and will only matter if the zerothorder term, $-1+B / m$, is close to zero. If transmission is frequency-dependent $(c=0)$ and density-dependent mortality is small, then $B / m \approx R_{0}$, where $R_{0}$ is the basic reproductive number of the pathogen. So as $R_{0} \rightarrow 1$, for non-zero (but small) $\kappa$ the likelihood that sterilizing pathogens are favoured by frequency-dependent transmission increases, even if the population is strongly regulated by density-dependent fecundity and density-dependent mortality only has a weak effect.

\section{$5 \quad N$-strain model}

In a similar fashion to the derivation of (13), we can extend the model to include $N$ strains. Doing so gives

$$
\begin{aligned}
d x & \left.=\left[b(\mathbf{x})-d(\mathbf{x}) x-\sum_{j} \beta_{j}(\mathbf{x}) y_{j}\right)\right] d \tau+\Omega^{-1 / 2} M_{x} \\
d y_{i} & =\left[\left(\beta_{i}(\mathbf{x})-\mu(\mathbf{x})\right) y_{i}+\nu\left(\sum_{j} y_{j}-n y_{i}\right)\right] d \tau+\Omega^{-1 / 2} M_{y_{i}}, \quad i=1,2, \ldots, N
\end{aligned}
$$

where

$$
\begin{aligned}
& M_{x}=\sqrt{(b(\mathbf{x})+d(\mathbf{x})) x} d W_{1}-\sum_{j} \sqrt{\beta_{j}(\mathbf{x}) y_{j}} d \tilde{W}_{j} \\
& M_{y_{i}}=\sqrt{\mu(\mathbf{x}) y_{i}} d W_{i+1}+\sqrt{\beta_{i}(\mathbf{x}) y_{i}} d \tilde{W}_{i}-\sum_{j} \sqrt{\nu y_{i}} d \hat{W}_{i j}+\sum_{j} \sqrt{\nu y_{j}} d \hat{W}_{j i} .
\end{aligned}
$$

Note that $d W_{i}, d \tilde{W}_{i}$ and $d \hat{W}_{i j}$ are all Wiener processes; we have just separated them for notational clarity. Thus $d \mathbf{W}$ is a $(n+1) \times 1$ vector whose $i$-th entry is $d W_{i}, d \tilde{\mathbf{W}}$ is a $n \times 1$ vector whose $i$-th entry is $d \tilde{W}_{i}$, and $d \hat{\mathbf{W}}$ is a $N \times N$ matrix whose $i, j$-th entry is $d \hat{W}_{i j}$. System (35) is then simulated using Euler-Maruyama method (Allen 2011). The reason that we have relied upon Euler-Maruyama method to simulate the system given by (35) rather than using Gillespie's algorithm (Gillespie 1977) to simulate the full stochastic process from which (35) is derived is due to computational requirements. The code used to simulate the system using Gillespie's algorithm and Euler-Maruyama method can be found with the Supplementary Information.

Although in (35) we have assumed each type mutates to any other type with equal probability (random mutation), we may ask what happens when each type can only mutate to its neighbouring phenotypes with equal probability (single-step mutation). For purposes of comparison, we will assume that the single-step mutation scheme has the same total 
mutation rate as the random mutation scheme. For the random mutation scheme, each type $i$ can mutate to any type $j$ at rate $\nu$, so there are $\nu(N-1)$ possible $j$ that $i$ mutates to and there are $N$ possible $i$. Thus the total mutation rate is $\nu N(N-1)$. For single-step mutations, there are $N-2$ 'interior' phenotypes, which can mutate in two directions, and then there are the two boundary phenotypes, $\delta=0$ and $\delta=1$, which can only mutate in one direction (into the interior). Thus the total number of mutations in the single-step scheme is $2(N-2)+1+1=2(N-1)$, and so the single-step scheme will have the same total rate of mutation as the random mutation scheme if the per capita rate a given phenotype will mutate to a specified phenotype is $\nu N(N-1) /[2(N-1)]=\nu N / 2$. In Figure 7 we plot simulation results showing a comparison between the random mutation and single-step mutation schemes. Since the two schemes deliver similar results (Fig. 7), in the paper we have relied upon the random mutation scheme.

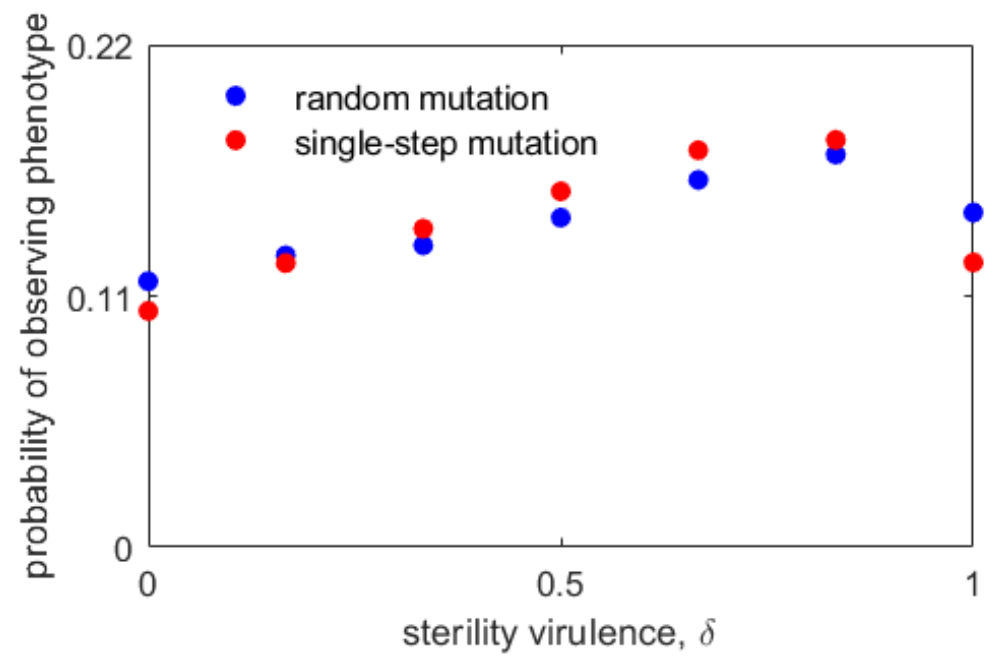

Figure 7: Comparison of the stationary distributions between the model where phenotypes mutate to any other phenotype with equal probability (random mutation; blue circles) and the model where phenotypes mutate to neighbouring phenotypes with equal probability (single-step mutation; red circles). The circles are $2.5 \times 10^{4}$ simulations of the model (35) with $N=7$ and $b(\mathbf{x})=\tilde{b}\left(x+\sum_{i}\left[1-\delta_{i}\right] y_{i}\right), d(\mathbf{x})=m+\kappa\left(x+\sum_{i} y_{i}\right), \mu(\mathbf{x})=\alpha+d(\mathbf{x})$, and $\beta(\mathbf{x})=$ $\frac{B x}{1+q\left(x+\sum_{i} y_{i}\right)}$. Parameter values are $\{B, \alpha, m, \kappa, \tilde{b}, \nu, \Omega\}=\left\{16.8,0.05,0.75,0.3,4,10^{-6}, 10^{4}\right\}$, with $q=3.125$.

This article is protected by copyright. All rights reserved. 